\title{
Benefits and harms of medical cannabis: a scoping review of systematic reviews
}

Misty Pratt ${ }^{1}$, Adrienne Stevens ${ }^{1,2}$, Micere Thuku' ${ }^{1}$, Claire Butler ${ }^{1,3}$, Becky Skidmore ${ }^{4}$, L. Susan Wieland ${ }^{5}$, Mark Clemons ${ }^{6,7}$, Salmaan Kanji, ${ }^{6,8,9}$ and Brian Hutton ${ }^{1,6^{*}}$ (D)

\begin{abstract}
Background: There has been increased interest in the role of cannabis for treating medical conditions. The availability of different cannabis-based products can make the side effects of exposure unpredictable. We sought to conduct a scoping review of systematic reviews assessing benefits and harms of cannabis-based medicines for any condition.
\end{abstract}

Methods: A protocol was followed throughout the conduct of this scoping review. A protocol-guided scoping review conduct. Searches of bibliographic databases (e.g., MEDLINE ${ }^{\circledR}$ Embase, PsycINFO, the Cochrane Library) and gray literature were performed. Two people selected and charted data from systematic reviews. Categorizations emerged during data synthesis. The reporting of results from systematic reviews was performed at a high level appropriate for a scoping review.

Results: After screening 1975 citations, 72 systematic reviews were included. The reviews covered many conditions, the most common being pain management. Several reviews focused on management of pain as a symptom of conditions such as multiple sclerosis (MS), injury, and cancer. After pain, the most common symptoms treated were spasticity in MS, movement disturbances, nausea/vomiting, and mental health symptoms. An assessment of review findings lends to the understanding that, although in a small number of reviews results showed a benefit for reducing pain, the analysis approach and reporting in other reviews was sub-optimal, making it difficult to know how consistent findings are when considering pain in general. Adverse effects were reported in most reviews comparing cannabis with placebo $(49 / 59,83 \%)$ and in $20 / 24$ (83\%) of the reviews comparing cannabis to active drugs. Minor adverse effects (e.g., drowsiness, dizziness) were common and reported in over half of the reviews. Serious harms were not as common, but were reported in 21/59 (36\%) reviews that reported on adverse effects. Overall, safety data was generally reported study-by-study, with few reviews synthesizing data. Only one review was rated as high quality, while the remaining were rated of moderate $(n=36)$ or low/critically low $(n=35)$ quality.

Conclusions: Results from the included reviews were mixed, with most reporting an inability to draw conclusions due to inconsistent findings and a lack of rigorous evidence. Mild harms were frequently reported, and it is possible the harms of cannabis-based medicines may outweigh benefits.

Systematic review registration: The protocol for this scoping review was posted in the Open Access (https://ruor. uottawa.ca/handle/10393/37247).

Keywords: Cannabis, Marijuana, Medical marijuana, Scoping review, Systematic review

\footnotetext{
* Correspondence: bhutton@ohri.ca

${ }^{1}$ Knowledge Synthesis Group, Ottawa Methods Centre, Ottawa Hospital

Research Institute, The Ottawa Hospital, General Campus, 501 Smyth Road,

Ottawa, Ontario K1H 8 L6, Canada

${ }^{6}$ School of Epidemiology and Public Health, University of Ottawa, 451 Smyth

Road, Ottawa, Ontario K1H 8 M5, Canada

Full list of author information is available at the end of the article
}

(c) The Author(s). 2019 Open Access This article is distributed under the terms of the Creative Commons Attribution 4.0 International License (http://creativecommons.org/licenses/by/4.0/), which permits unrestricted use, distribution, and

reproduction in any medium, provided you give appropriate credit to the original author(s) and the source, provide a link to the Creative Commons license, and indicate if changes were made. The Creative Commons Public Domain Dedication waiver (http://creativecommons.org/publicdomain/zero/1.0/) applies to the data made available in this article, unless otherwise stated. 


\section{Background}

Interest in medical applications of marijuana (Cannabis sativa) has increased dramatically during the past 20 years. A 1999 report from the National Academies of Sciences, Engineering, and Medicine supported the use of marijuana in medicine, leading to a number of regulatory medical colleges providing recommendations for its prescription to patients [1]. An updated report in 2017 called for a national research agenda, improvement of research quality, improvement in data collection and surveillance efforts, and strategies for addressing barriers in advancing the cannabis agenda [2].

Proponents of medical cannabis support its use for a highly varied range of medical conditions, most notably in the fields of pain management [3] and multiple sclerosis [4]. Marijuana can be consumed by patients in a variety of ways including smoking, vaporizing, ingesting, or administering sublingually or rectally. The plant consists of more than 100 known cannabinoids, the main ones of relevance to medical applications being tetrahydrocannabinol (THC) and cannabidiol (CBD) [5]. Synthetic forms of marijuana such as dronabinol and nabilone are also available as prescriptions in the USA and Canada [6].

Over the last decade, there has been an increased interest in the use of medical cannabis products in North America. It is estimated that over 3.5 million people in the USA are legally using medical marijuana, and a total of USD $\$ 6.7$ billion was spent in North America on legal marijuana in 2016 [7]. The number of Canadian residents with prescriptions to purchase medical marijuana from Health Canada-approved growers tripled from 30,537 in 2015 to near 100,000 in 2016 [8]. With the legalization of recreational-use marijuana in parts of the USA and in Canada in October 2018, the number of patients using marijuana for therapeutic purposes may become more difficult to track. The likely increase in the numbers of individuals consuming cannabis also necessitates a greater awareness of its potential benefits and harms.

Plant-based and plant-derived cannabis products are not monitored as more traditional medicines are, thereby increasing the uncertainty regarding its potential health risks to patients [3]. While synthetic forms of cannabis are available by prescription, different cannabis plants and products contain varied concentrations of THC and CBD, making the effects of exposure unpredictable [9]. While short-lasting side effects including drowsiness, loss of short-term memory, and dizziness are relatively well known and may be considered minor, other possible effects (e.g., psychosis, paranoia, anxiety, infection, withdrawal) may be more harmful to patients.
There remains a considerable degree of clinical equipoise as to the benefits and harms of marijuana use for medical purposes [10-13]. To understand the extent of synthesized evidence underlying this issue, we conducted a scoping review [14] of systematic reviews evaluating the benefits and/or harms of cannabis (plant-based, plant-derived, and synthetic forms) for any medical condition. We located and mapped systematic reviews to summarize research that is available for consideration for practice or policy questions in relation to medical marijuana.

\section{Methods}

A scoping review protocol was prepared and posted to the University of Ottawa Health Sciences Library's online repository (https://ruor.uottawa.ca/handle/10393/3 7247). We used the PRISMA for Scoping Reviews checklist to guide the reporting of this report (see Additional file 1) [15].

\section{Literature search and process of study selection}

An experienced medical information specialist developed and tested the search strategy using an iterative process in consultation with the review team. Another senior information specialist peer-reviewed the strategy prior to execution using the PRESS Checklist [16]. We searched seven Ovid databases: MEDLINE ${ }^{\oplus}$, including Epub Ahead of Print and In-Process \& Other Non-Indexed Citations, Embase, Allied and Complementary Medicine Database, PsycINFO, the Cochrane Database of Systematic Reviews, the Database of Abstracts of Reviews of Effects, and the Health Technology Assessment Database. The final peer-reviewed search strategy for MEDLINE was translated to the other databases (see Additional file 2). We performed the searches on November 3, 2017.

The search strategy incorporated controlled vocabulary (e.g., "Cannabis," "Cannabinoids," "Medical Marijuana") and keywords (e.g., "marijuana," "hashish," "tetrahydrocannabinol") and applied a broad systematic review filter where applicable. Vocabulary and syntax were adjusted across the databases and where possible animal-only and opinion pieces were removed, from the search results.

Gray literature searching was limited to relevant drug and mental health databases, as well as HTA (Health Technology Assessment) and systematic review databases. Searching was guided by the Canadian Agency for Drugs and Technologies in Health's $(\mathrm{CADTH})$ checklist for health-related gray literature (see Additional file 3). We performed searches between January and February 2018. Reference lists of overviews were searched for relevant systematic 
reviews, and we searched for full-text publications of abstracts or protocols.

Management of all screening was performed using Distiller SR Software - (Evidence Partners Inc., Ottawa, Canada). Citations from the literature search were collated and de-duplicated in Reference Manager (Thomson Reuters: Reference Manager 12 [Computer Program]. New York: Thomson Reuters 2011), and then uploaded to Distiller. The review team used Distiller for Levels 1 (titles and abstracts) and 2 (fulltext) screening. Pilot testing of screening questions for both levels were completed prior to implementation. All titles and abstracts were screened in duplicate by two independent reviewers (MT and MP) using the liberal accelerated method [17]. This method requires only one reviewer to assess an abstract as eligible for full-text screening, and requires two reviewers to deem the abstract irrelevant. Two independent reviewers (MT and MP) assessed full- text reports for eligibility. Disagreements during fulltext screening were resolved through consensus, or by a third team member (AS). The process of review selection was summarized using a PRISMA flow diagram (Fig. 1) [18].

\section{Review selection criteria}

English-language systematic reviews were included if they reported that they investigated harms and/or benefits of medical or therapeutic use of cannabis for adults and children for any indication. Definitions related to medical cannabis/marijuana are provided in Table 1 . We also included synthetic cannabis products, which are prescribed medicines with specified doses of THC and CBD. Reviews of solely observational designs were included only in relation to adverse effects data, in order to focus on the most robust evidence available. We considered studies to be systematic reviews if at least one database was

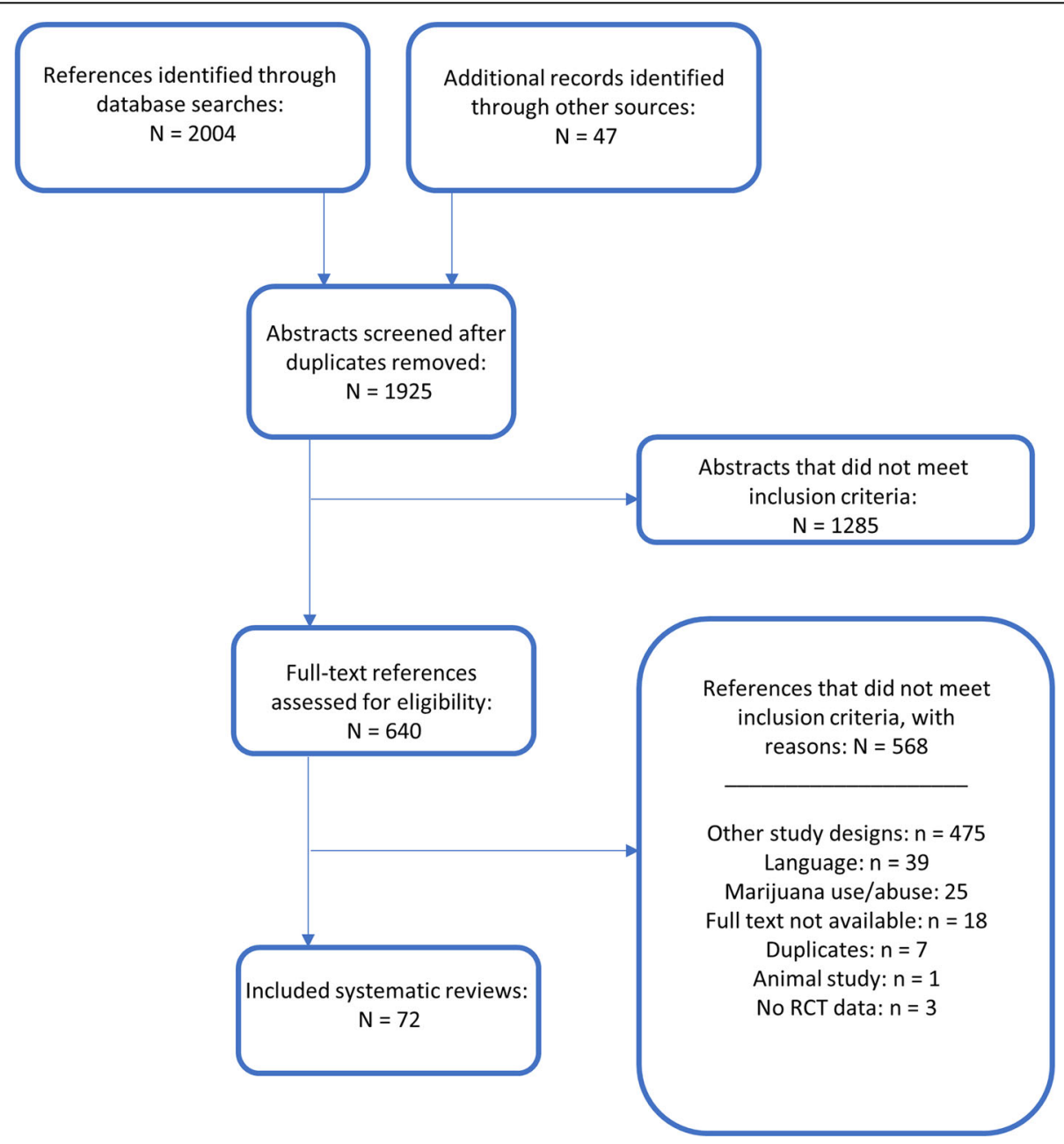

Fig. 1 PRISMA-style flow diagram of the review selection process 
Table 1 Context for the use of cannabis-related terms during the review selection process

\begin{tabular}{ll}
\hline Term & Definition \\
\hline $\begin{array}{l}\text { Medical marijuana } \\
\text { (or marijuana for medical use) }\end{array}$ & $\begin{array}{l}\text { The term medical marijuana refers to using the whole, unprocessed marijuana plant or its basic extracts to } \\
\text { treat symptoms of illness and other conditions (https://www.drugabuse.gov/publications/drugfacts/marijuana- } \\
\text { medicine) } \\
\text { Whether marijuana is recognized as medicine varies from country to country. The US Food and Drug } \\
\text { Administration (FDA) has not recognized or approved the marijuana plant as medicine, but a growing number } \\
\text { of states have legalized marijuana for medical use. In Canada, it is legal to possess cannabis for medical purposes, } \\
\text { and legalization for non-medical use is set to take place in } 2018 .\end{array}$
\end{tabular}

searched with search dates reported, at least one eligibility criterion was reported, the authors had assessed the quality of included studies, and there was a narrative or quantitative synthesis of the evidence. Reviews assessing multiple interventions (both pharmacological and complementary and alternative medicine (CAM) interventions) were included if the data for marijuana studies was reported separately. Published and unpublished guidelines were included if they conducted a systematic review encompassing the criteria listed above.

We excluded overviews of systematic reviews, reviews in abstract form only, and review protocols. We further excluded systematic reviews focusing on recreational, accidental, acute, or general cannabis use/abuse and interventions such as synthetic cannabinoids not approved for therapeutic use (e.g., K2 or Spice).

\section{Data collection and quality assessment}

All data were collected electronically in a pre-developed form using Microsoft Excel software (Microsoft Corporation, Seattle, USA). The form was pilot tested on three included reviews by three people. One reviewer (MP or $\mathrm{CB}$ ) independently extracted all data, and a second reviewer (MT) verified all of the items collected and checked for any omitted data. Disagreements were resolved by consensus and consultation with a third reviewer if necessary. A data extraction form with the list of included variables is provided in Additional file 4. All collected data has also been made available in the online supplemental materials associated with this report.

Quality assessment of systematic reviews was performed using the AMSTAR-2 [20] tool. One reviewer (MP or CB) independently assessed quality, while a second reviewer (MT) verified the assessments. Disagreements were resolved by consensus and consultation with a third reviewer if necessary. The tool consists of 16 items in total, with four critical domains and 12 non-critical domains. The AMSTAR-2 tool is not intended to generate an overall score, and instead allows for an overall rating based on weaknesses in critical domains. Reviews were rated as high (no critical flaws with zero or one non-critical flaw), moderate (no critical flaws with $\geq 1$ non-critical flaw), low (one critical flaw with/without non-critical weakness), or critically low ( $>1$ critical flaw with/without non-critical weakness) quality.

\section{Evidence synthesis}

We used a directed content analytic approach [21] with an initial deductive framework [22] that allowed flexibility for inductive analysis if refinement or development of new categorization was needed. The framework used to categorize outcome data results is outlined in Table 2. Where reviews had a mix of

Table 2 Outcome result categorization

\begin{tabular}{ll}
\hline Outcome data categorization & Definition \\
\hline Favors intervention & $\begin{array}{l}\text { Review authors conducted a meta-analysis and/or narrative synthesis (i.e., count data) which shows a benefi } \\
\text { cial effect for cannabis. } \\
\text { Favors control }\end{array}$ \\
$\begin{array}{ll}\text { Review authors conducted a meta-analysis and/or narrative synthesis (i.e., count data) which shows a } \\
\text { beneficial effect of control. }\end{array}$ \\
$\begin{array}{l}\text { Review authors do not provide enough information to make a clear conclusion, or state that data from } \\
\text { included studies is insufficient. } \\
\text { between groups }\end{array}$ & $\begin{array}{l}\text { Review authors conducted a meta-analysis and/or narrative synthesis (i.e., count data) which shows no } \\
\text { significant difference between cannabis and control groups (precision with confidence interval [included null } \\
\text { results] preferred over } p \text { value). } \\
\text { Reported study-by-study (SBS) }\end{array} \quad \begin{array}{l}\text { Authors narratively review the primary study data, without providing an overall count of positive, negative, } \\
\text { or no difference effects. Included studies are reviewed individually, with or without author's final } \\
\text { recommendations and conclusions }\end{array}$ \\
\hline
\end{tabular}


narrative and quantitative data, results from metaanalyses were prioritized over count data or study-bystudy data. The extraction and reporting of data results was performed at a high level and did not involve an in-depth evaluation, which is appropriate for a scoping review [14]. Review authors' conclusions and/or recommendations were extracted and reported narratively.

\section{Changes from the study protocol}

For feasibility, we decided to limit the inclusion of systematic reviews of only observational study designs to those that addressed adverse events data. All other steps of the review were performed as planned.

\section{Results}

\section{Search findings}

The PRISMA flow diagram describing the process of review selection is presented in Fig. 1. After duplicates were removed, the search identified a total of 1925 titles and abstracts, of which 47 references were located through the gray literature search. Of the total 1925 citations assessed during Level 1 screening, 1285 were deemed irrelevant. We reviewed full-text reports for the 640 reviews of potential relevance, and of these, 567 were subsequently excluded, leaving a total of 72 systematic reviews that were included; the associated data collected are provided in Additional file 5. A listing of the reports excluded during full-text review is provided in Additional file 6.

\section{Characteristics of included reviews}

There were 63 systematic reviews $[4,19,23-83]$ and nine guidelines with systematic reviews [84-92]. Overall, 27 reviews were performed by researchers in Europe, 16 in the USA, 15 in Canada, eight in Australia, two in Brazil, and one each in Israel, Singapore, South Africa, and China. Funding was not reported in $29(40 \%)$ of the reviews, and the remaining reviews received funding from non-profit or academic $(n=20 ; 28 \%)$, government $(n=14$; $19 \%)$, industry $(n=3 ; 4 \%)$, and mixed $(n=1 ; 1 \%)$ sources. Five reviews reported that they did not receive any funding for the systematic review. Tables 3 , $4,5,6,7,8,9,10,11,12$, and 13 provide an overview of the characteristics of the 72 included systematic reviews.

The reviews were published between 2000 and 2018 (median year 2014), and almost half (47\%) were focused solely on medical cannabis. Four (6\%) reviews covered both medical and other cannabis use (recreational and substance abuse), 19 (26\%) reported multiple pharmaceutical interventions (cannabis being one), six (8\%) reported various CAM interventions (cannabis being one), and nine (13\%) were mixed pharmaceutical and CAM interventions (cannabis being one). Multiple databases were searched by almost all of the reviews (97\%), with Medline/PubMed or Embase common to all.

\section{Cannabis use}

Figure 2 illustrates the different cannabis-based interventions covered by the included reviews. Plant-based cannabis consists of whole plant products such as marijuana or hashish. Plant-derived cannabinoids are active constituents of the cannabis plant, such as tetrahydrocannabinol (THC), cannabidiol (CBD), or a combination of THC:CBD (also called nabiximols, under the brand name Sativex) [3]. Synthetic cannabinoids are manufactured rather than extracted from the plant and include drugs such as nabilone and dronabinol.

Twenty-seven reviews included solely interventions from plant-derived cannabinoids, 10 studied solely synthetic cannabinoids, and eight included solely studies on plant-based cannabis products. Twentyfour reviews covered a combination of different types of cannabis, and the remaining three systematic reviews did not report which type of cannabinoid was administered in the included studies.

\section{Population}

The systematic reviews covered a wide range of conditions and illnesses, the most notable being pain management. Seventeen reviews looked at specific types of pain including neuropathic [31, 42, 62, 69, 85, 90], chronic [26, 32, 52, 58, 80], cancer [84, 87], non-cancer [41, 68], and acute [38] types of pain (one review covered all types of pain) [65]. Twenty-seven reviews (38\%) also focused on management of pain as a symptom of conditions such as multiple sclerosis (MS) $[6,23,27,43,46,52,63,85,92]$, injury $[29,35$, $36,69]$, cancer $[37,43,65,88]$, inflammatory bowel disease (IBD) [28], rheumatic disease (RD) [49, 51, 73], diabetes [68-70], and HIV [48, 53, 67]. In Fig. 3, the types of illnesses addressed by the set of included reviews are graphically represented, with overlap between various conditions and pain. Some systematic reviews covered multiple diseases, and therefore the total number of conditions represented in Fig. 3 is greater than the total number of included reviews.

One review included a pediatric-only population, in the evaluation of marijuana for nausea and vomiting following chemotherapy [54]. Although trials in both adult and child populations were eligible for thirteen (18\%) reviews, only two additional reviews included 


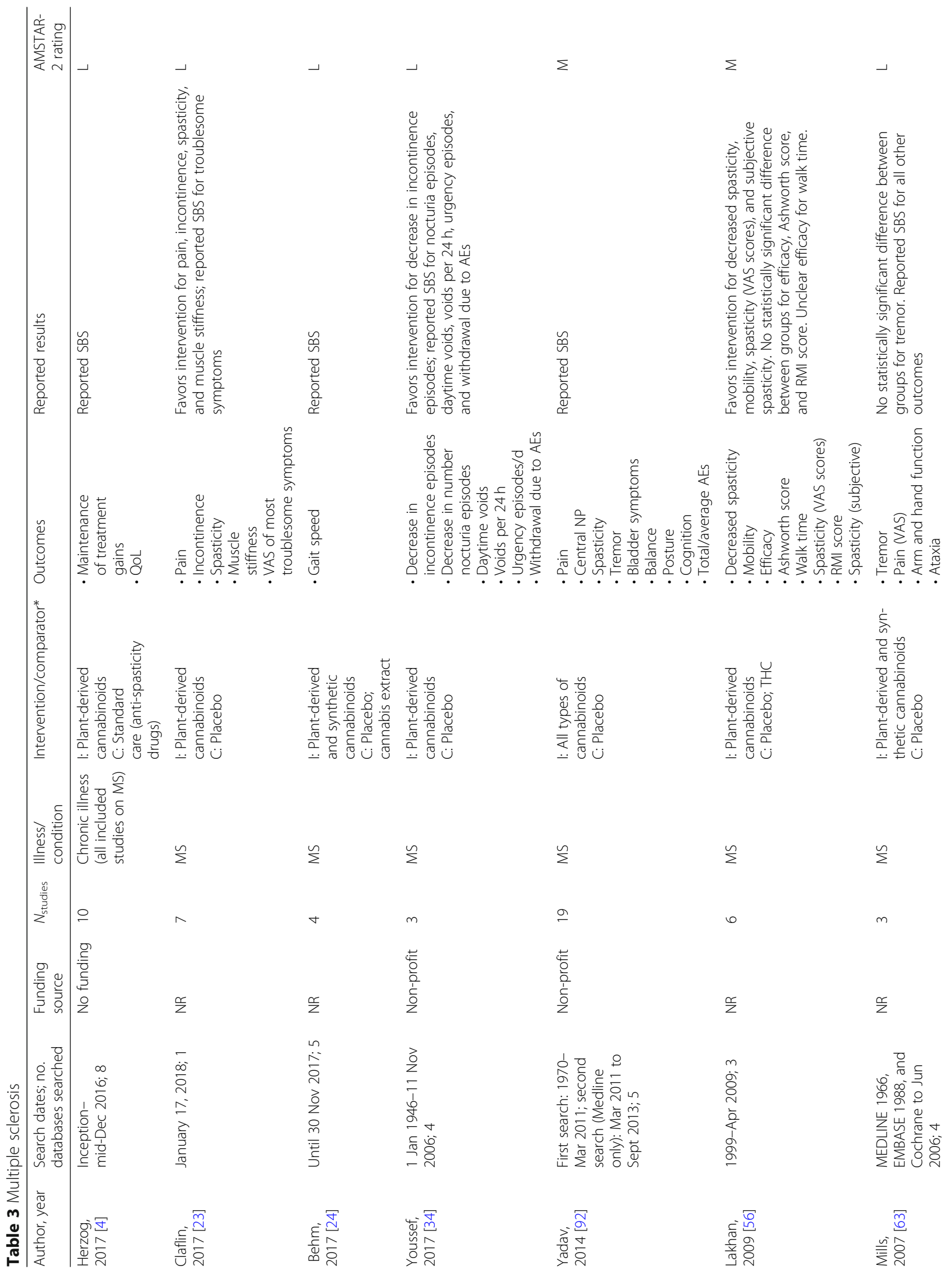




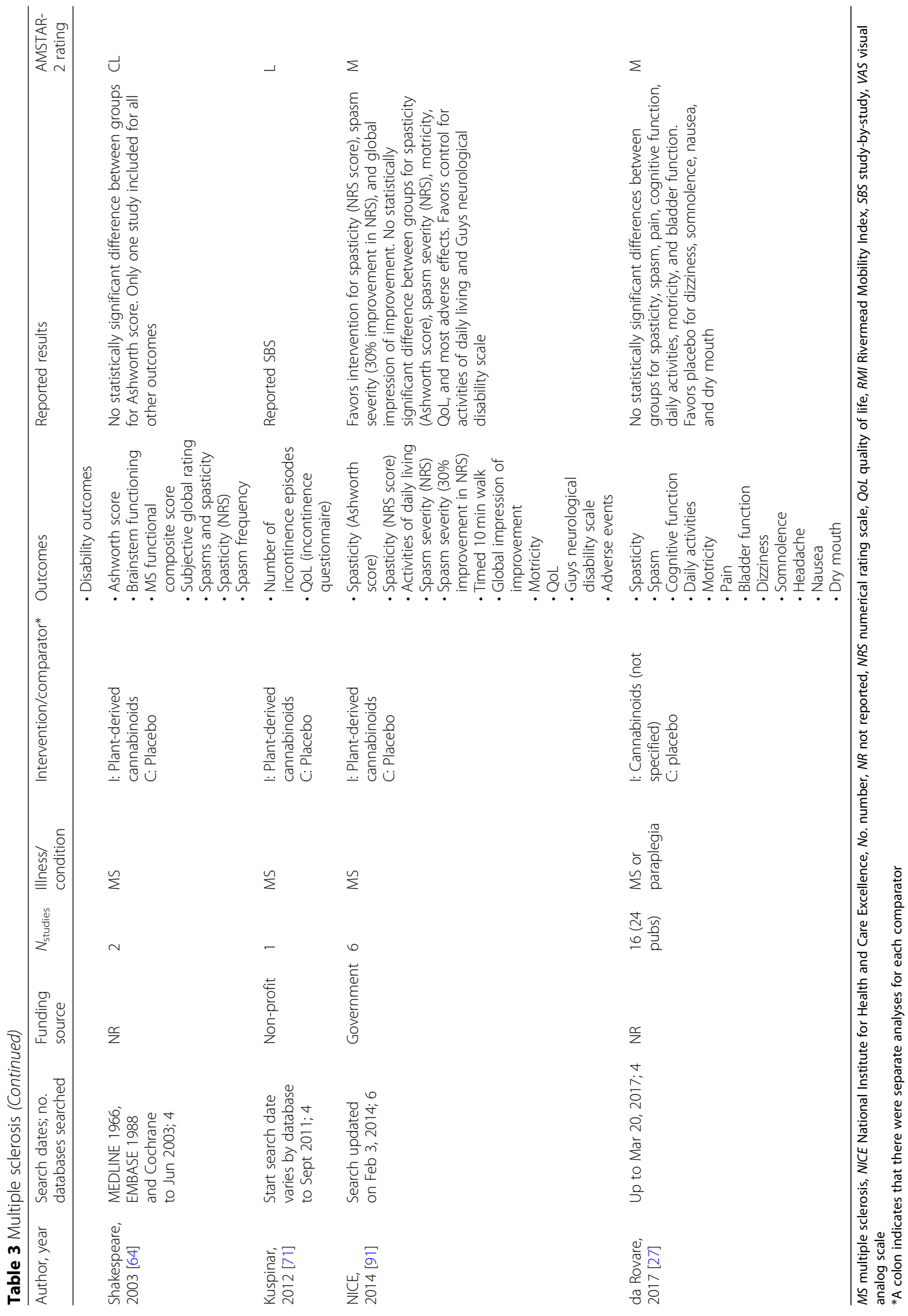




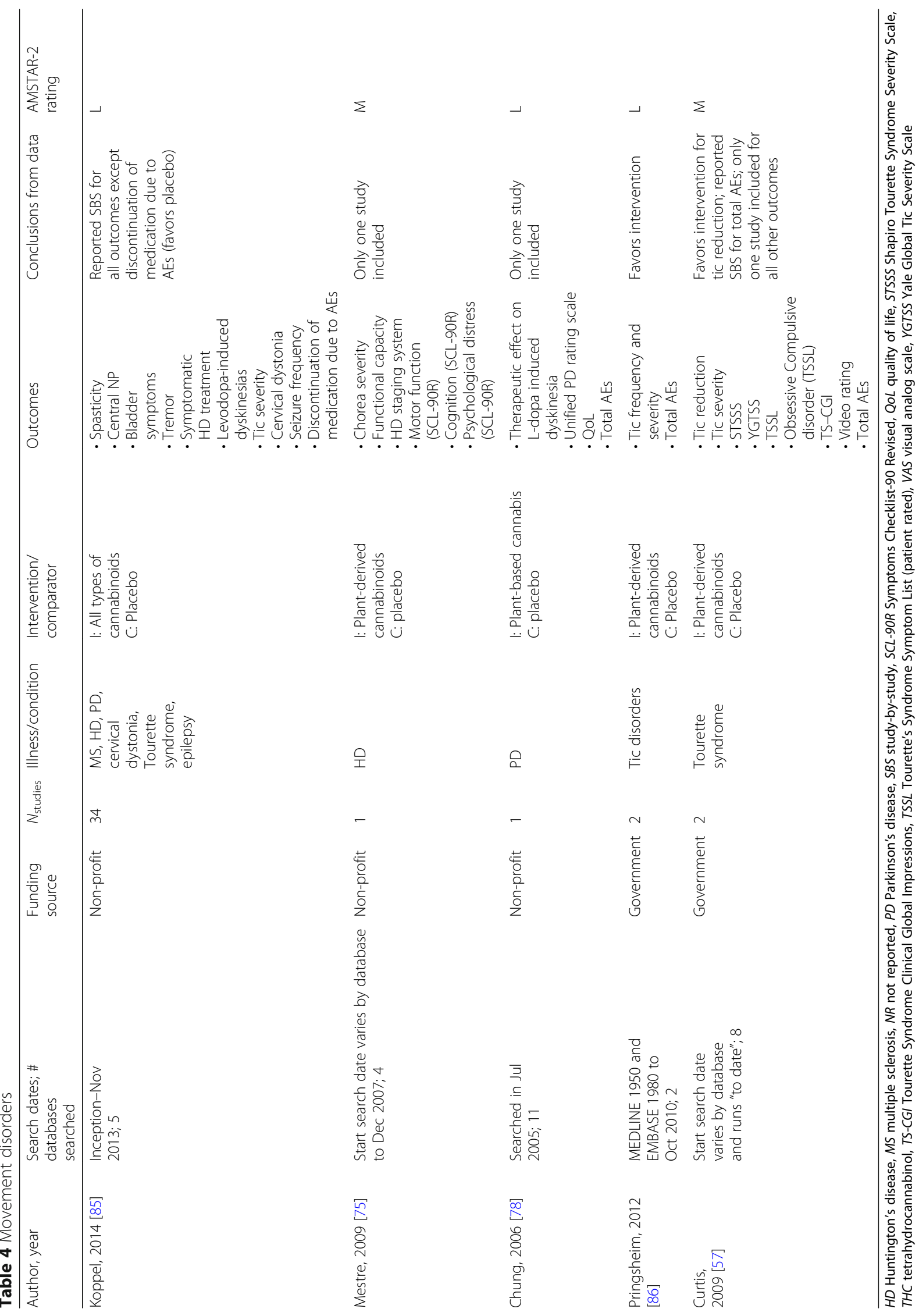




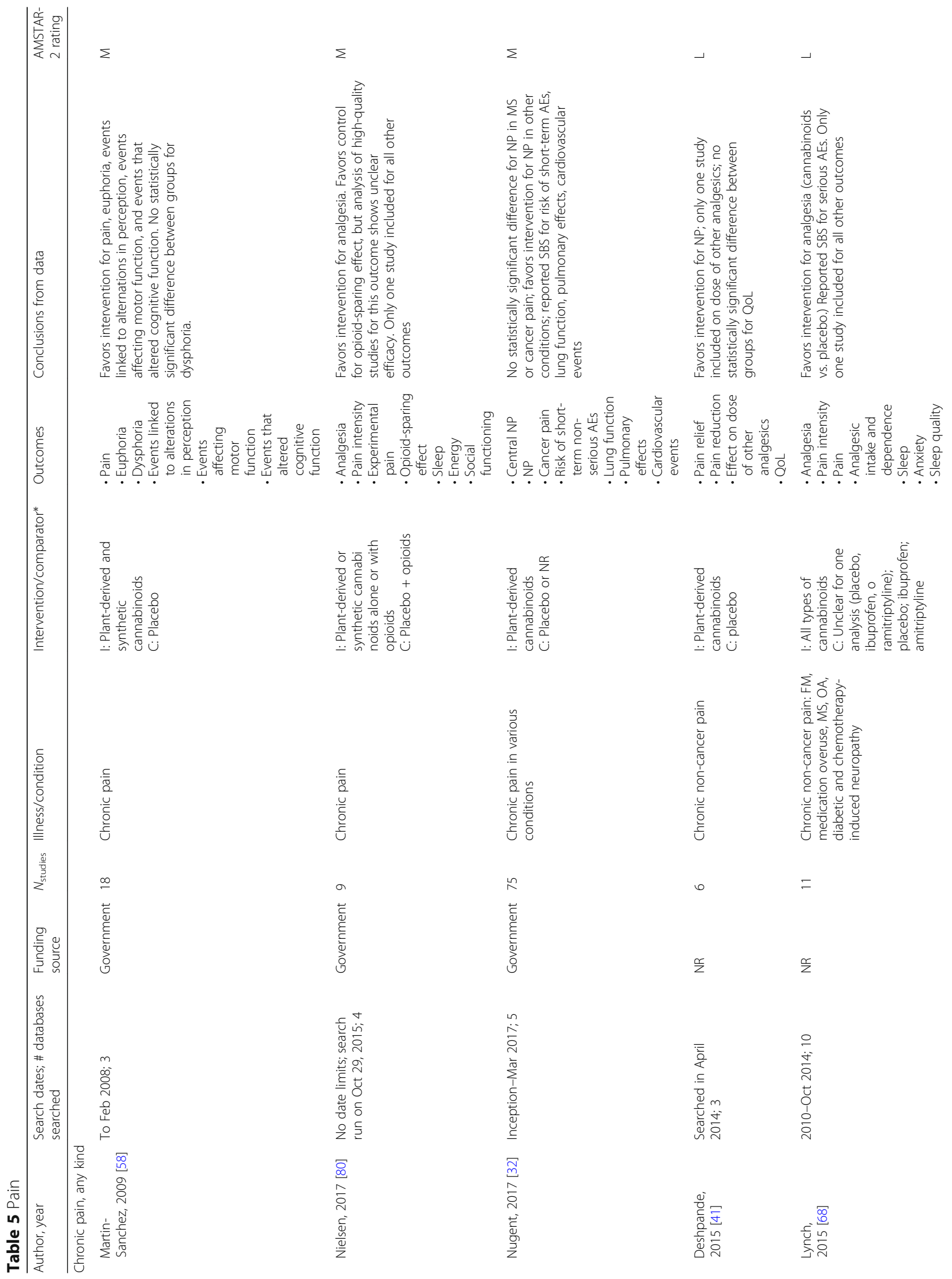




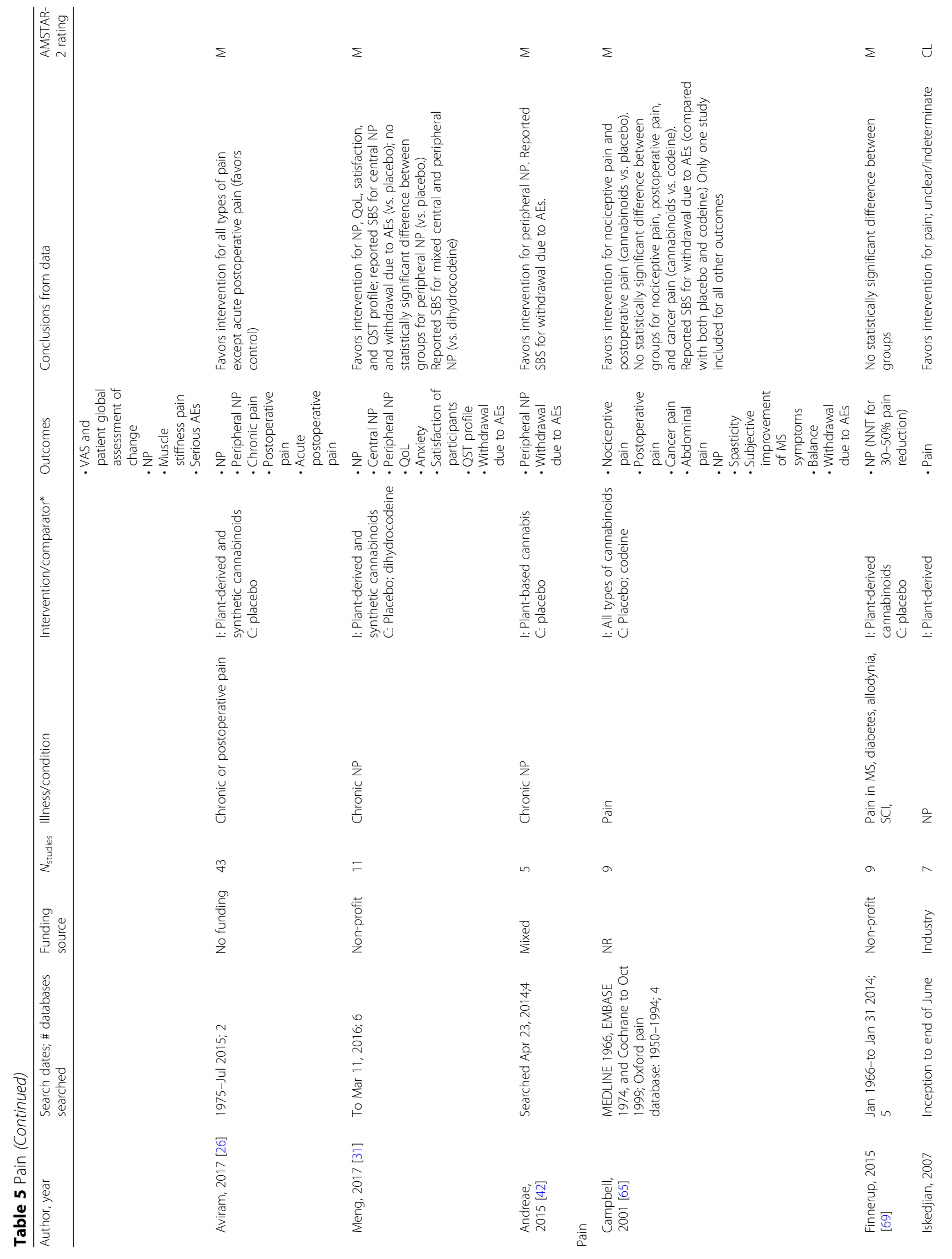




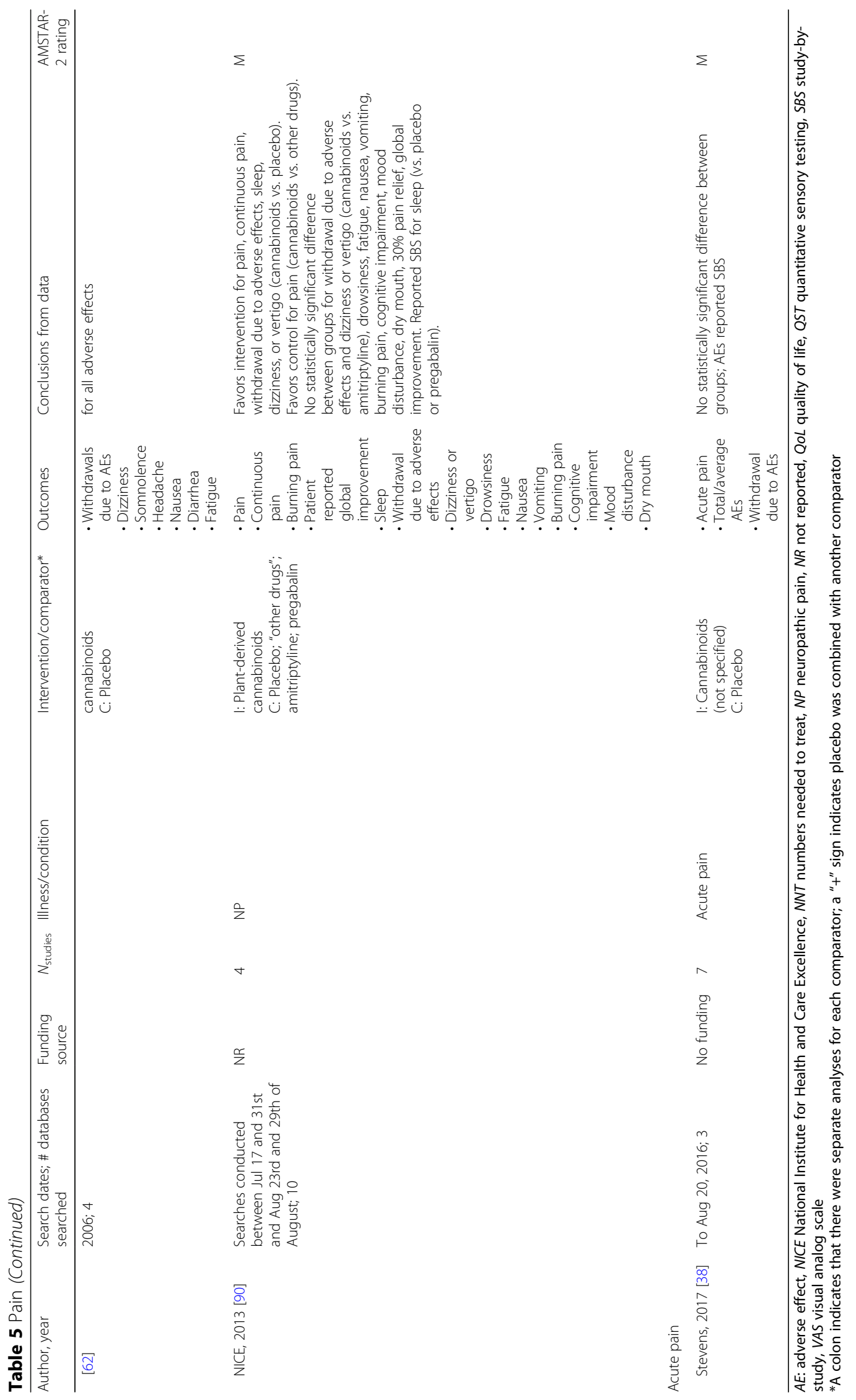




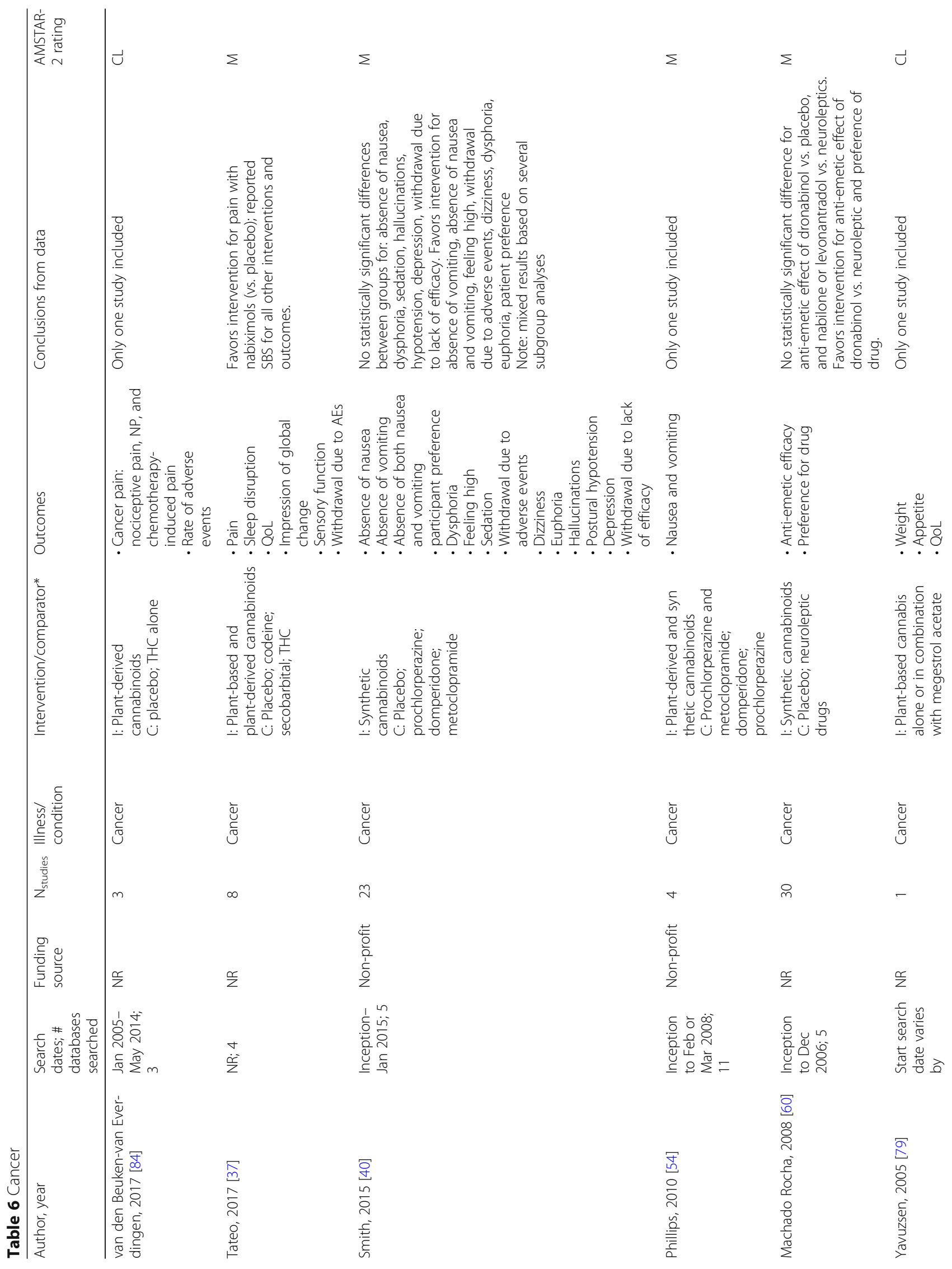




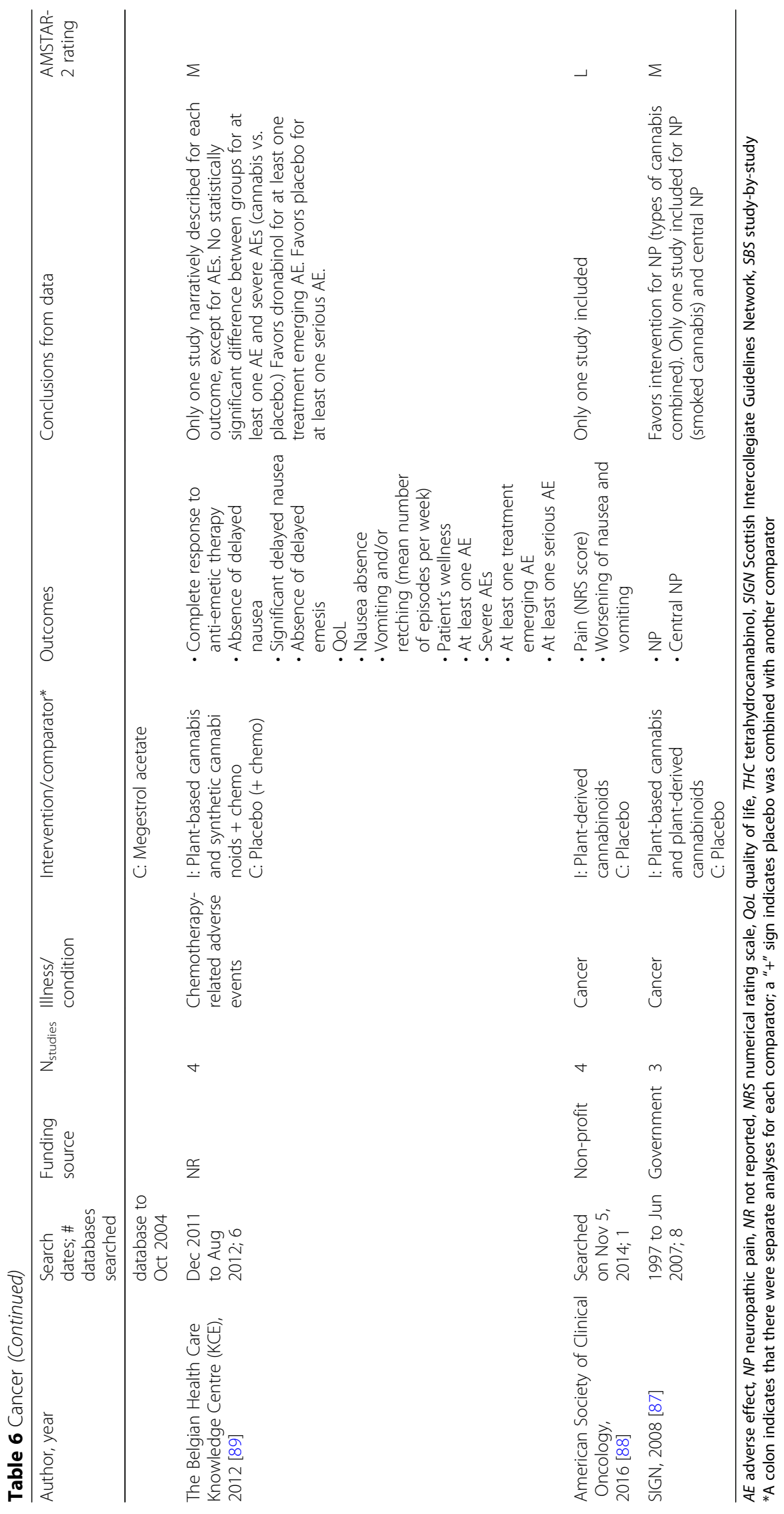




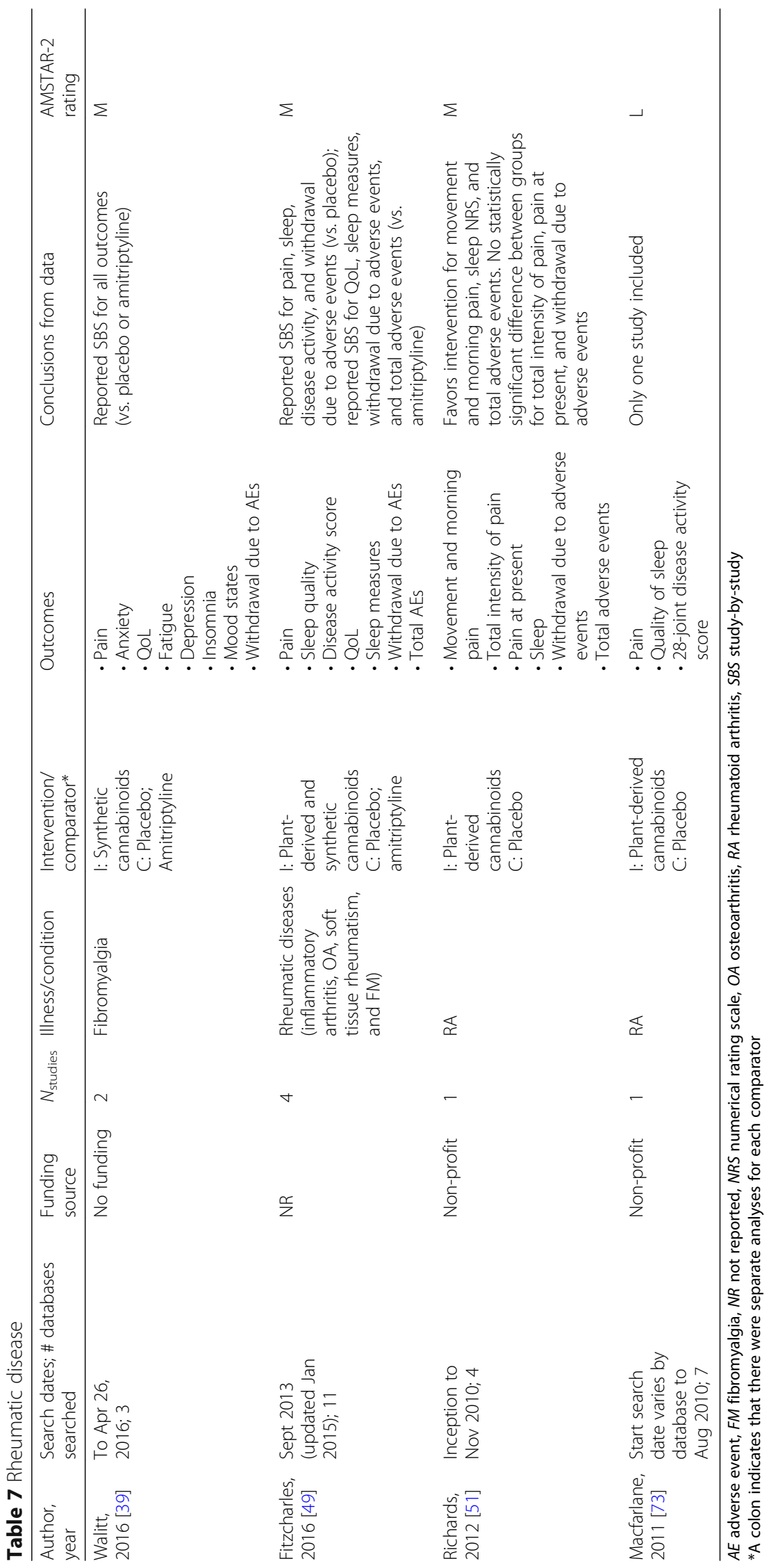


Table 8 Injury

\begin{tabular}{|c|c|c|c|c|c|c|c|c|}
\hline $\begin{array}{l}\text { Author, } \\
\text { year \{refid\} }\end{array}$ & $\begin{array}{l}\text { Search dates; \# } \\
\text { databases searched }\end{array}$ & $\begin{array}{l}\text { Funding } \\
\text { source }\end{array}$ & $N_{\text {studies }}$ & $\begin{array}{l}\text { Illness/ } \\
\text { condition }\end{array}$ & $\begin{array}{l}\text { Intervention/ } \\
\text { comparator }\end{array}$ & Outcomes & Conclusions from data & $\begin{array}{l}\text { AMSTAR- } \\
2 \text { rating }\end{array}$ \\
\hline $\begin{array}{l}\text { Snedecor, } \\
2013 \text { [29] }\end{array}$ & To Dec 2011; 5 & Industry & 1 & $\begin{array}{l}\text { NP associated } \\
\text { with spinal } \\
\text { cord injury }\end{array}$ & $\begin{array}{l}\text { I: Synthetic } \\
\text { cannabinoids } \\
\text { C: Placebo }\end{array}$ & $\begin{array}{l}\text { - NP } \\
\cdot \text { All-cause } \\
\text { discontinuation }\end{array}$ & $\begin{array}{l}\text { Favors control for NP; } \\
\text { no statistically significant } \\
\text { different between groups } \\
\text { for all-cause discontinuation }\end{array}$ & $\mathrm{CL}$ \\
\hline $\begin{array}{l}\text { Mehta, } \\
2016 \text { [35] }\end{array}$ & 2009-Sept 2015; 4 & NR & 2 & $\begin{array}{l}\text { Spinal cord } \\
\text { injury }\end{array}$ & $\begin{array}{l}\text { I: Plant-derived and } \\
\text { Synthetic } \\
\text { cannabinoids } \\
\text { C: } \\
\text { diphenhydramine }\end{array}$ & $\begin{array}{l}\text { - NP } \\
\text { - Spastic pain }\end{array}$ & Reported SBS & L \\
\hline $\begin{array}{l}\text { Meyer, } \\
2010[55]\end{array}$ & 1980-2008; 4 & NR & 2 & $\begin{array}{l}\text { Acquired } \\
\text { brain injury }\end{array}$ & $\begin{array}{l}\text { I: Synthetic } \\
\text { cannabinoids } \\
\text { C: Placebo }\end{array}$ & $\begin{array}{l}\text { - Intercranial } \\
\text { pressure } \\
\text { - Glasgow } \\
\text { outcome scale } \\
\text { - Disability rating } \\
\text { scale } \\
\text { - Mortality rates } \\
\text { - Activities of daily } \\
\text { living } \\
\text { - QoL }\end{array}$ & Reported SBS & M \\
\hline $\begin{array}{l}\text { Wheaton, } \\
2009 \text { [76] }\end{array}$ & $\begin{array}{l}\text { Jan } 1980 \text { to May } \\
2008 ; 2\end{array}$ & $\begin{array}{l}\text { Non- } \\
\text { profit }\end{array}$ & 2 & $\begin{array}{l}\text { Traumatic } \\
\text { brain injury }\end{array}$ & $\begin{array}{l}\text { I: Synthetic } \\
\text { cannabinoids } \\
\text { C: placebo }\end{array}$ & $\begin{array}{l}\text { - Global outcome } \\
\text { score ( } 3 \text { and } 6 \\
\text { months) }\end{array}$ & $\begin{array}{l}\text { No statistically significant } \\
\text { difference between groups }\end{array}$ & $\mathrm{CL}$ \\
\hline
\end{tabular}

$N P$ neuropathic pain, $N R$ not reported, QoL quality of life, SBS study-by-study

studies in children; these reviews evaluated cannabis in cancer [60] and a variety of conditions [25]. Many of the reviews $(n=25,35 \%)$ included only adults $\geq$ 18 years of age. Almost half of the reviews $(n=33$, $46 \%$ ) did not report a specific population for inclusion.

Cannabis was prescribed for a wide range of medical issues. The indication for cannabis use is illustrated in Fig. 4. Pain management $(n=27)$ was the most common indication for cannabis use. A number of reviews sought to address multiple disease symptoms $(n=12)$ or explored a more holistic treatment for the disease itself $(n=11)$. After pain, the most common symptoms being treated with cannabis were spasticity in MS, movement disturbances (such as dyskinesia, tics, and spasms), weight or nausea/vomiting, and mental health symptoms.

Figure 5 summarizes the breadth of outcomes analyzed in the included reviews. The most commonly addressed outcomes were withdrawal due to adverse effects, "other pain," neuropathic pain, spasticity, and the global impression of the change in clinical status. Many outcomes were reported using a variety of measures across reviews. For example, spasticity was measured both objectively (using the Ashworth scale) and subjectively (using a visual analog scale [VAS] or numerical rating scale [NRS]). Similarily, outcomes for pain included VAS or NRS scales, reduction in pain, pain relief, analgesia, pain intensity, and patient assessment of change in pain.

Table 9 Mental health

\begin{tabular}{|c|c|c|c|c|c|c|c|c|}
\hline $\begin{array}{l}\text { Author, year } \\
\text { \{refid\} }\end{array}$ & $\begin{array}{l}\text { Search dates; \# } \\
\text { databases } \\
\text { searched }\end{array}$ & $\begin{array}{l}\text { Funding } \\
\text { source }\end{array}$ & $N_{\text {studies }}$ & $\begin{array}{l}\text { Illness/ } \\
\text { condition }\end{array}$ & Intervention/comparator & Outcomes & $\begin{array}{l}\text { Conclusions from } \\
\text { data }\end{array}$ & $\begin{array}{l}\text { AMSTAR- } \\
2 \text { rating }\end{array}$ \\
\hline $\begin{array}{l}\text { Walsh, } \\
2017 \text { [19] }\end{array}$ & $\begin{array}{l}\text { 1960-Sept } \\
2015 ; 2\end{array}$ & Government & 31 & Mental health & $\begin{array}{l}\text { I: Plant-derived } \\
\text { cannabinoids } \\
\text { C: Cannabinoid with no } \\
\text { THC }\end{array}$ & $\begin{array}{l}\text { - Improvement in } \\
\text { anxiety and } \\
\text { depression }\end{array}$ & $\begin{array}{l}\text { Only one RCT } \\
\text { included (combined } \\
\text { with cross-sectional } \\
\text { data) }\end{array}$ & $\mathrm{CL}$ \\
\hline $\begin{array}{l}\text { O'Neil, } \\
2017 \text { [33] }\end{array}$ & $\begin{array}{l}\text { Inception-Mar } \\
2017 ; 6\end{array}$ & Government & 5 & PTSD & $\begin{array}{l}\text { I: All types of cannabinoids } \\
\text { C: Different dose or } \\
\text { different duration of dose } \\
\text { of cannabinoids }\end{array}$ & $\begin{array}{l}\text { N/A } \\
\text { observational } \\
\text { data }\end{array}$ & $\begin{array}{l}\text { N/A observational } \\
\text { data }\end{array}$ & M \\
\hline $\begin{array}{l}\text { McLoughlin, } \\
2014 \text { [45] }\end{array}$ & $\begin{array}{l}\text { To Aug 12, } \\
2013 ; 6\end{array}$ & Non-profit & 1 & Schizophrenia & $\begin{array}{l}\text { I: Plant-derived } \\
\text { cannabinoids } \\
\text { C: Amisulpride }\end{array}$ & $\begin{array}{l}\text { - Mental state } \\
\text { outcomes }\end{array}$ & $\begin{array}{l}\text { Only one study } \\
\text { included }\end{array}$ & $H$ \\
\hline
\end{tabular}




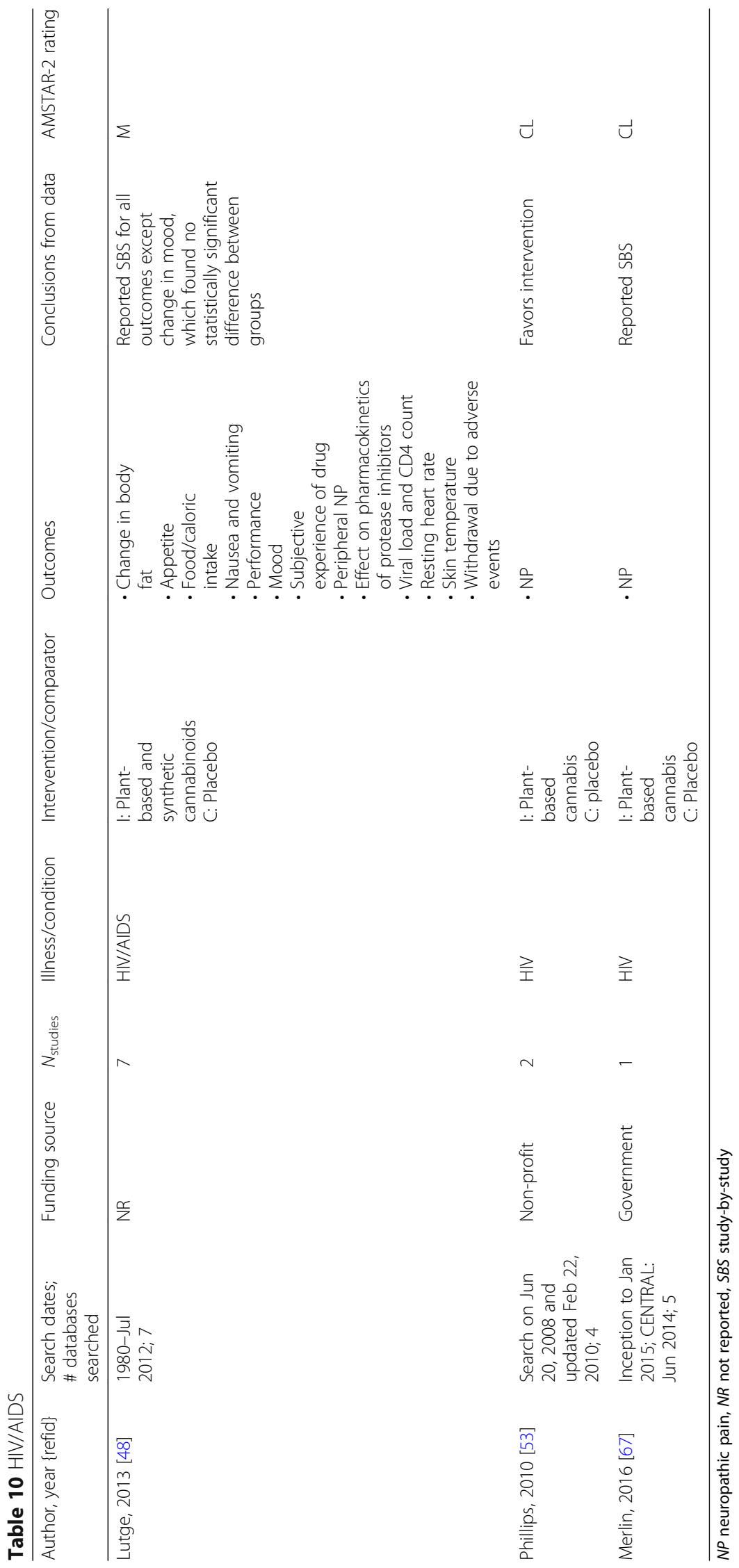




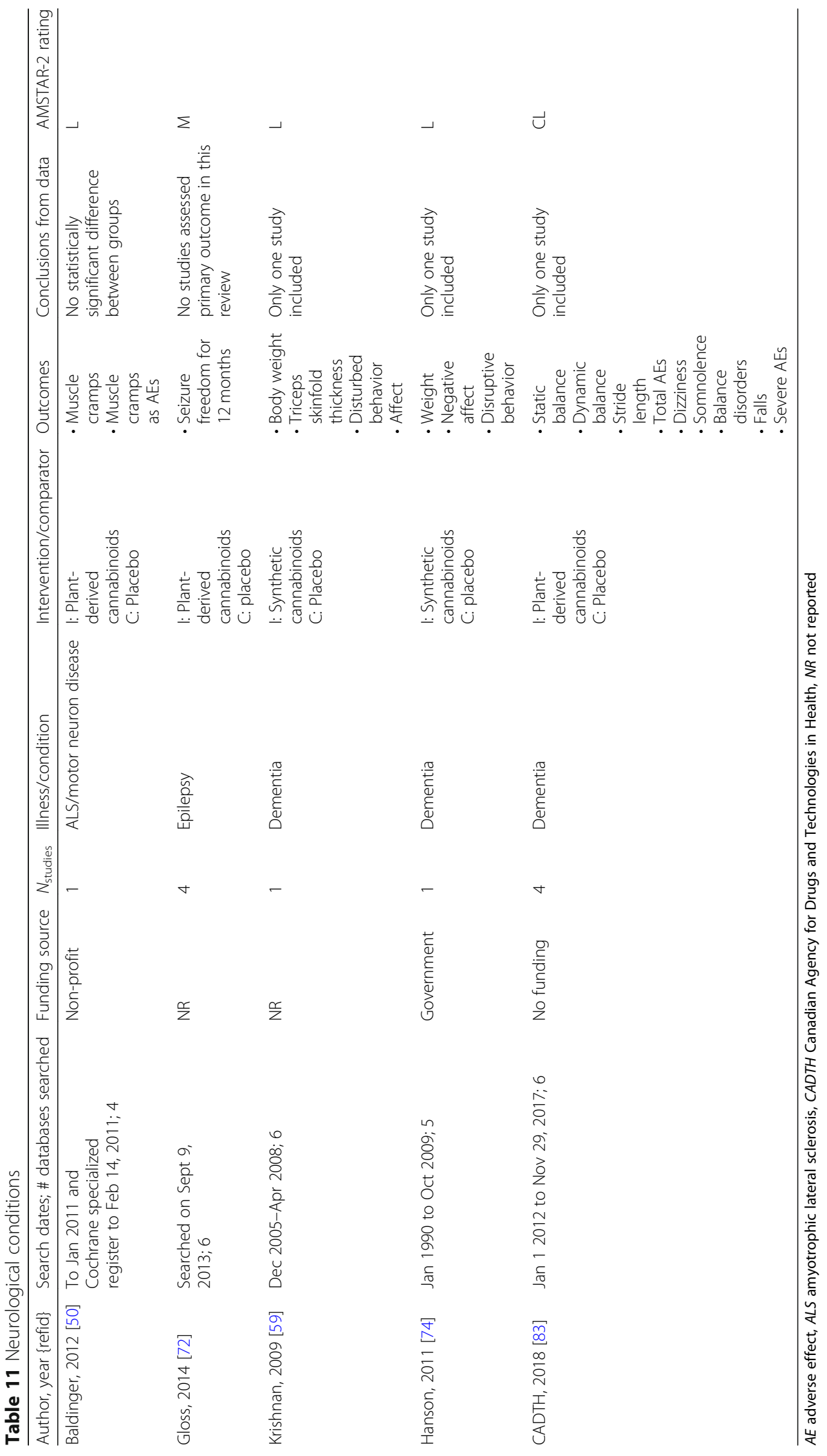




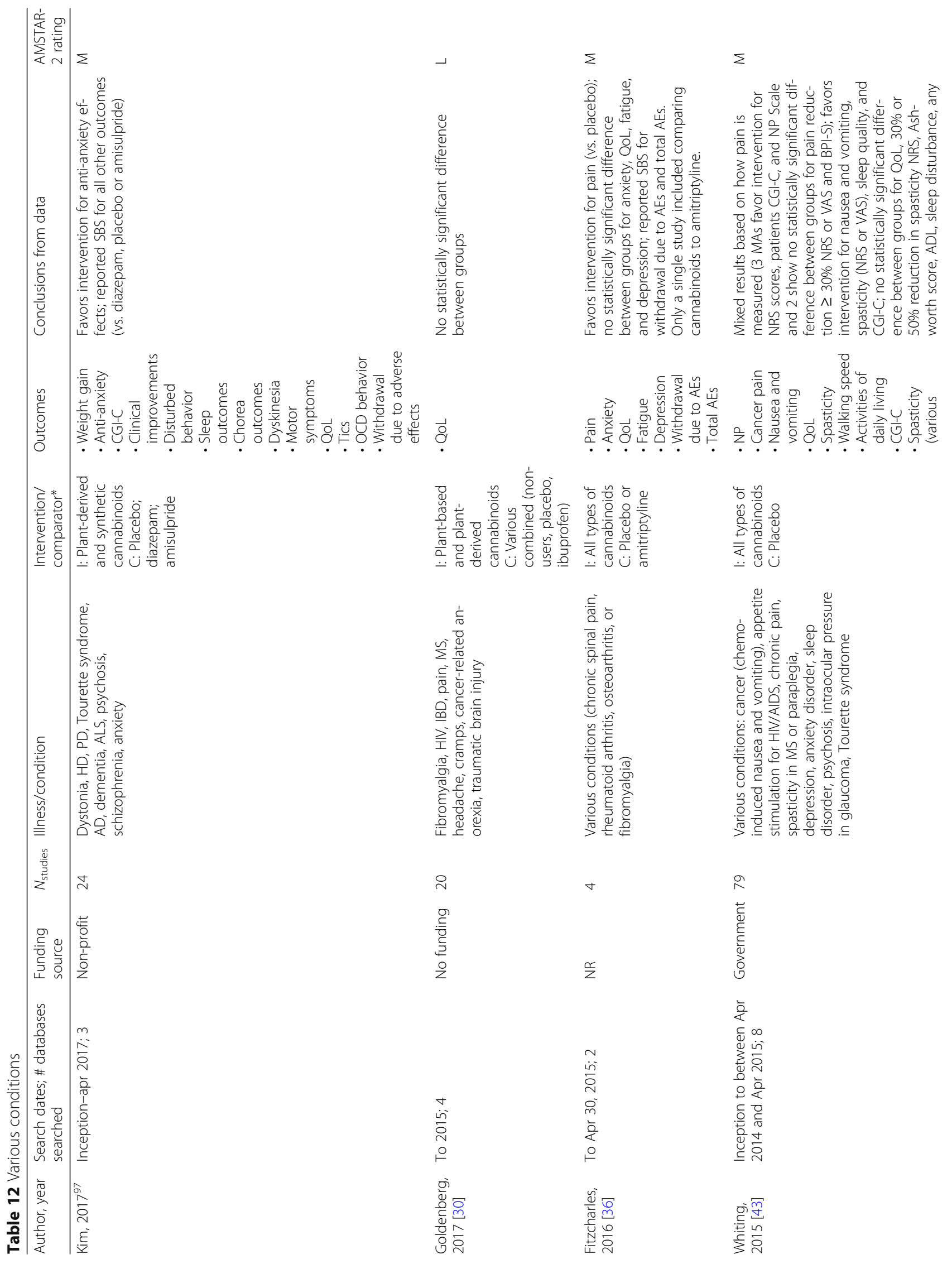


Pratt et al. Systematic Reviews

(2019) 8:320

Page 19 of 35

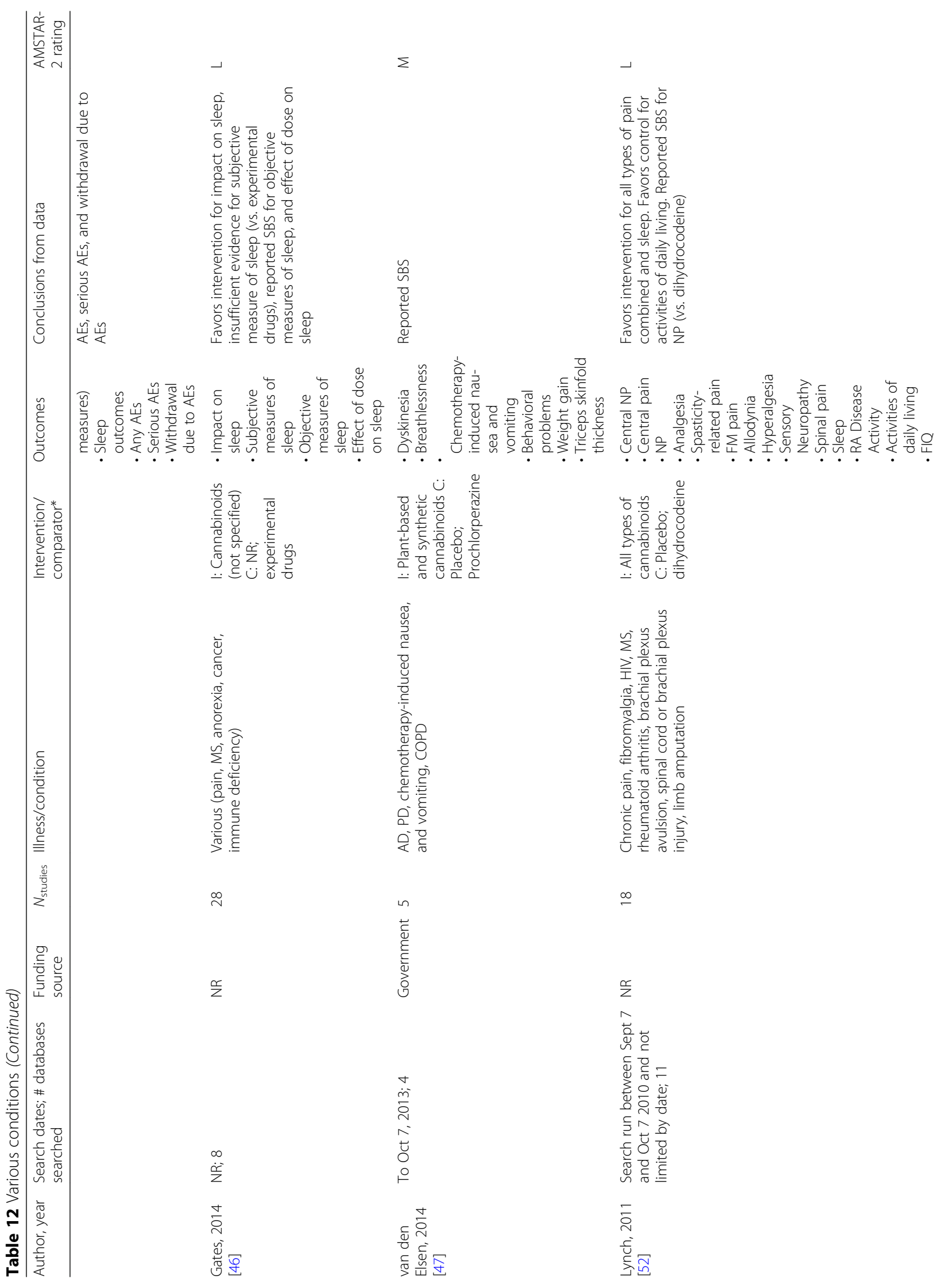




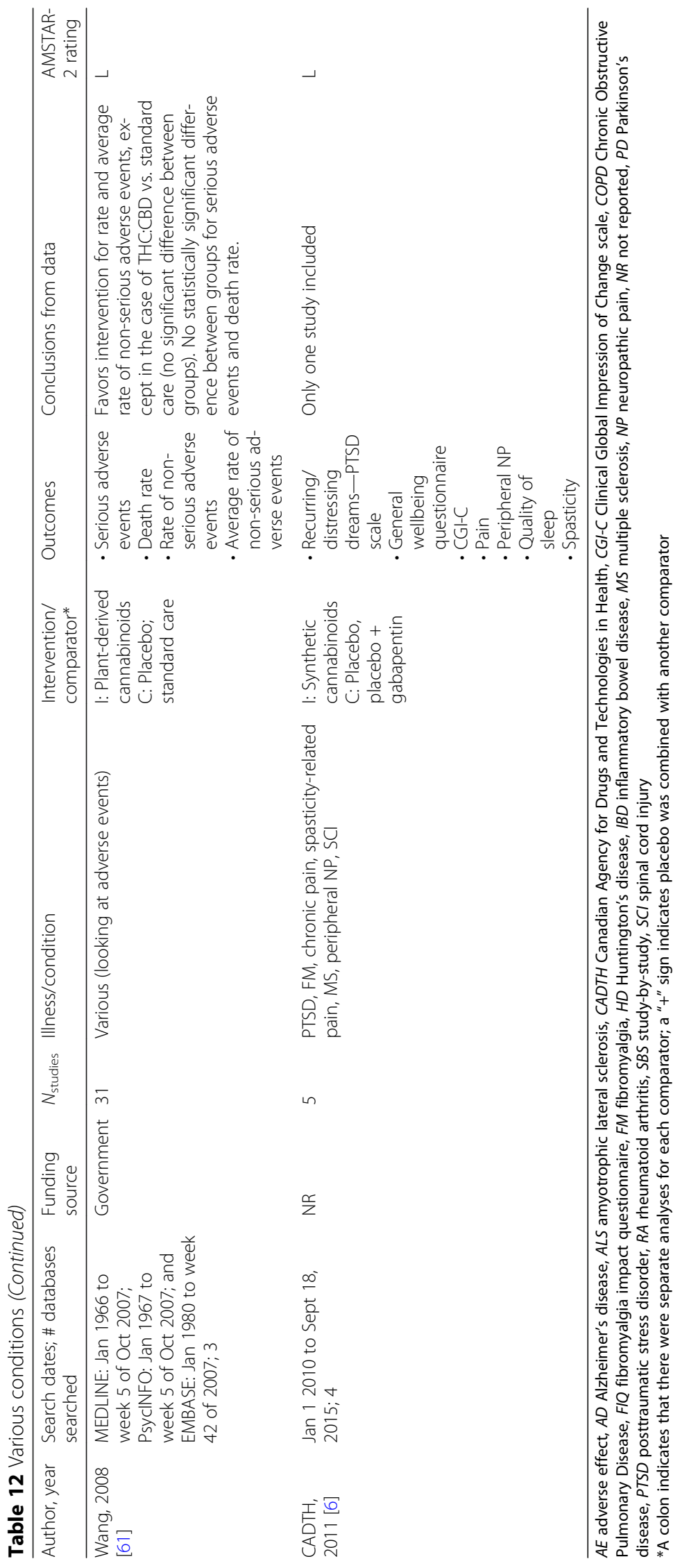




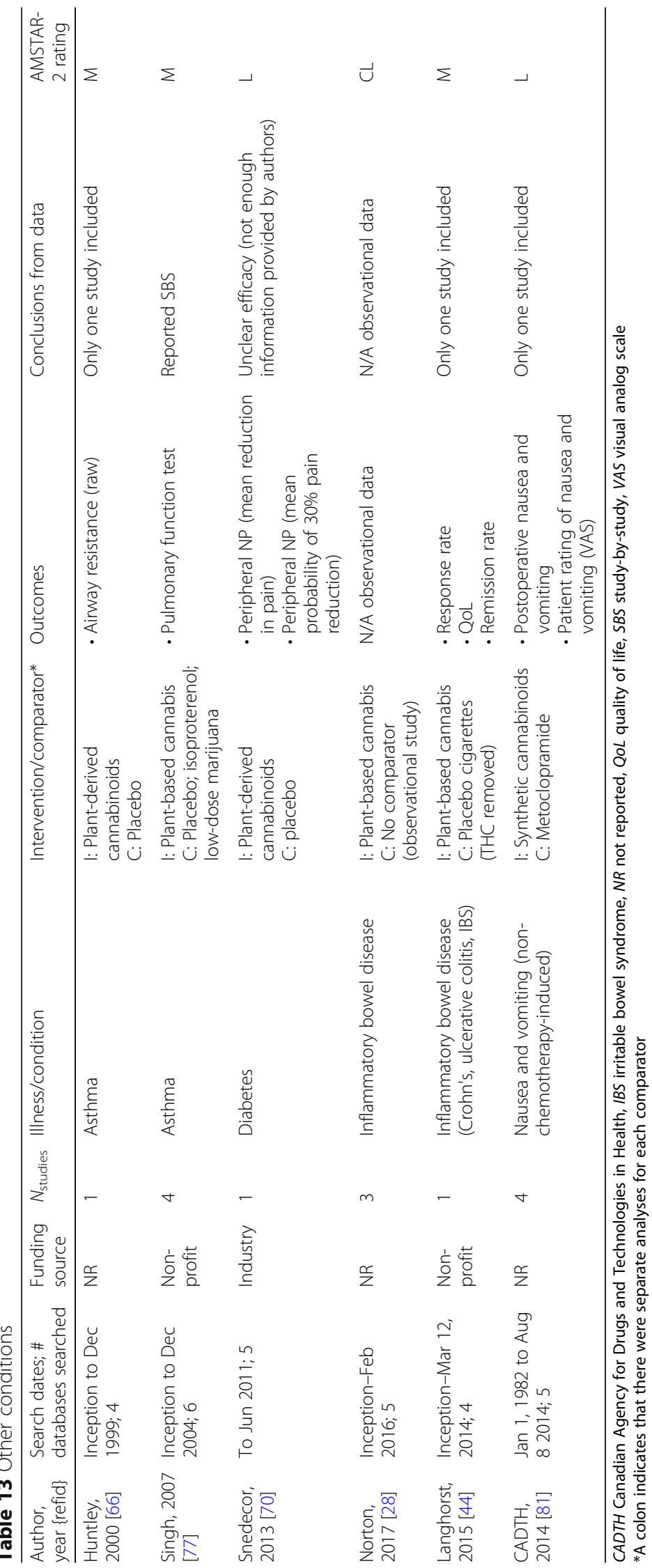




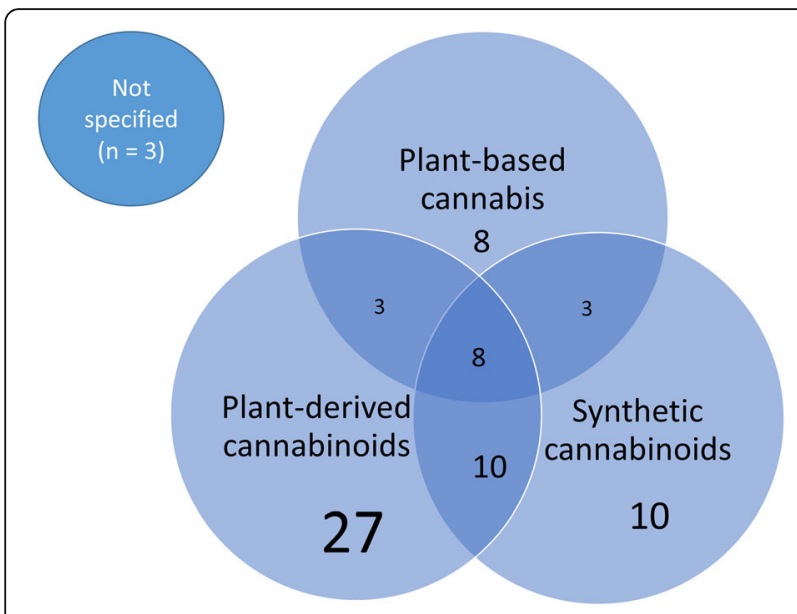

Fig. 2 Review coverage of the various cannabis-based interventions

\section{Quality of the systematic reviews}

Quality assessments of the included reviews based upon AMSTAR-2 are detailed in Additional file 7 and Additional file 8. Only one review was rated as high quality [45]. All other reviews were deemed to be of moderate $(n=36)$ or low/critically low $(n=35)$ methodological quality. Assessments for the domains deemed of critical importance for determining quality ratings are described below.

Only $20 \%$ of reviews used a comprehensive search strategy; another $47 \%$ were given a partial score because they had not searched the reference lists of the included reviews, trial registries, gray literature, and/ or the search date was older than 2 years. The remaining reviews did not report a comprehensive search strategy.
Over half of the reviews (51\%) used a satisfactory technique for assessing risk of bias (ROB) of the individual included studies, while $35 \%$ were partially satisfactory because they had not reported whether allocation sequence was truly random and/or they had not assessed selective reporting. The remaining reviews did not report a satisfactory technique for assessing ROB.

Most reviews (71\%) could not be assessed for an appropriate statistical method for combining results in a meta-analysis, as they synthesized study data narratively. Approximately $19 \%$ of reviews used an appropriate meta-analytical approach, leaving $10 \%$ that used inappropriate methods.

The final critical domain for the AMSTAR-2 determines whether review authors accounted for ROB in individual studies when discussing or interpreting the results of the review. The majority of reviews $(83 \%)$ did so in some capacity.

\section{Mapping results of included systematic reviews}

We mapped reviews according to authors' comparisons, the conditions or symptoms they were evaluating, and the categorization of the results (see Table 2). In some cases, reviews contributed to more than one comparison (e.g., cannabis versus placebo or active drug). As pain was the most commonly addressed outcome, we mapped this outcome separately from all other endpoints. This information is shown for all reviews and then restricted to reviews of moderate-to-high quality (as determined using the AMSTAR-2 criteria): cannabis versus placebo (Figs. 6 and 7), cannabis versus active drugs (Figs. 8 and 9), cannabis versus a combination of placebo and active drug (Figs. 10 and 11), one cannabis

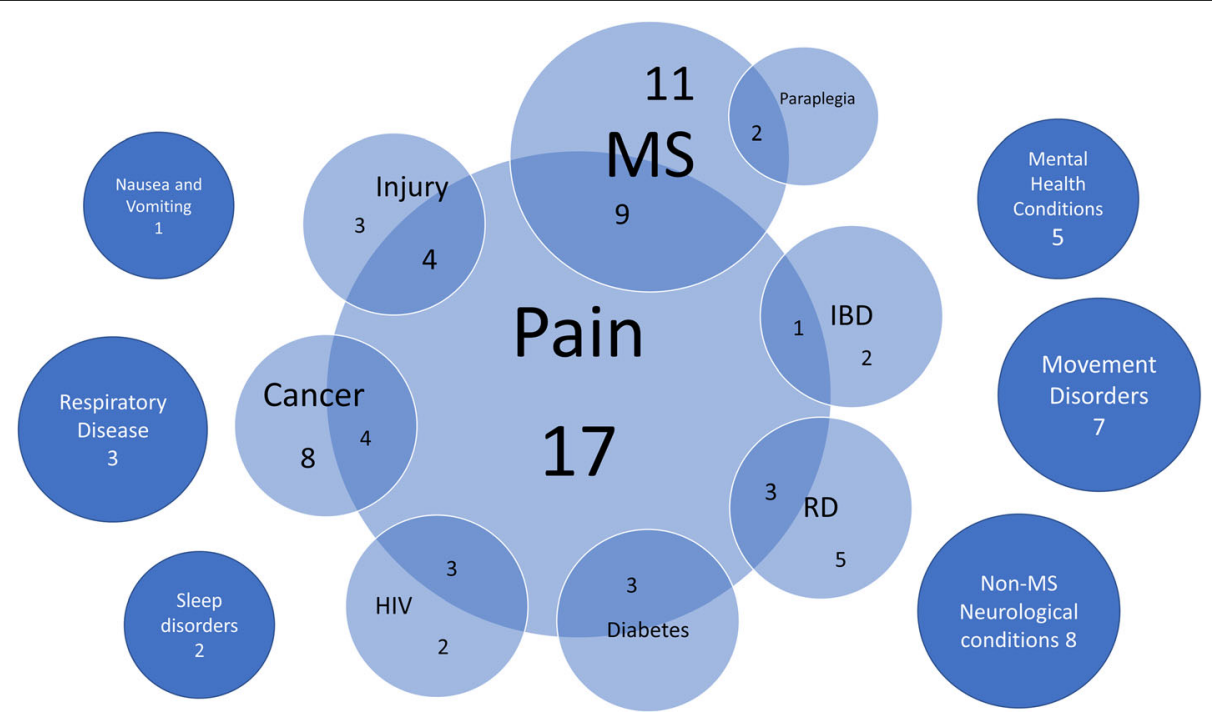

Fig. 3 Conditions or symptoms across reviews that were treated with cannabis. IBD inflammatory bowel disease, MS multiple sclerosis, RD rheumatic disease 


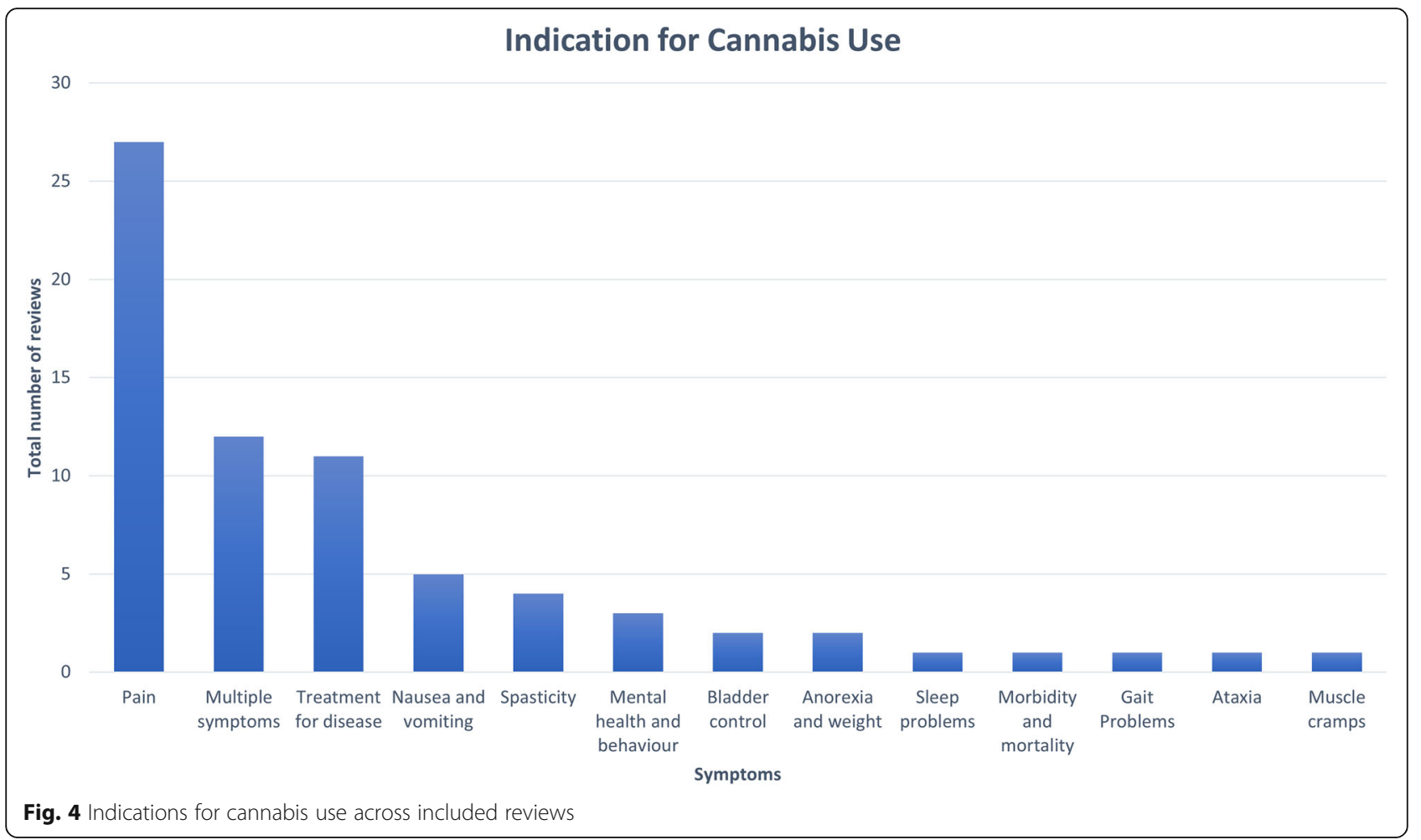

formulation versus other (Figs. 12 and 13), and cannabis analyzed against all other comparators (Fig. 14). Details on how to read the figures are provided in the corresponding figure legends. The median number of included studies across reviews was four, and ranged from one to seventy-nine (not shown in figures).

\section{Cannabis versus placebo}

Most reviews (59/72, 82\%) compared cannabis with placebo. Of these reviews, 34 (58\%) addressed pain outcomes and 47 (80\%) addressed non-pain outcomes, with most outcomes addressed by three reviews or fewer (Fig. 6). Some reviews had a mix of quantitative syntheses and study-by-study data reported (13/59, $22 \%)$, while another group of reviews (14/59, 24\%) only reported results study-by-study. Overall, 24\% $(14 / 59)$ of the cannabis versus placebo reviews had only one included study.

1. Pain outcomes

i. Reviews focused on addressing pain across conditions. In most cases, findings were discordant across reviews for the pain outcomes measured. For chronic non-cancer pain, however, two reviews favored cannabis over placebo for decreasing pain. One review assessing acute pain for postoperative pain relief found no difference between various cannabinoid medications and placebo. The distribution of findings was similar when restricting to moderate-tohigh-quality reviews.

ii. Reviews focused on treating a condition or family of related conditions. Various results were observed for pain. For MS and HIV/AIDS, one review each reported quantitative results favoring cannabis for decreased pain but with other reviews reporting results studyby-study, it is difficult to know, broadly, how consistent those findings are. For cancer, two reviews reported results favoring cannabis for decreased pain. For rheumatic disease, findings are discordant between two reviews, and another two reviews reported results studyby-study. One review that included studies of MS or paraplegia found no difference in pain between groups. For treating injury, one review showed that the placebo group had less pain and one review reported data study-bystudy. No reviews addressed pain in movement disorders, neurological conditions, and IBD.

For those reviews assessing pain as part of a focus on treating a range of conditions, two showed cannabis reduced pain $[43,52]$, but one showed mixed results depending on how pain was measured [43]. 


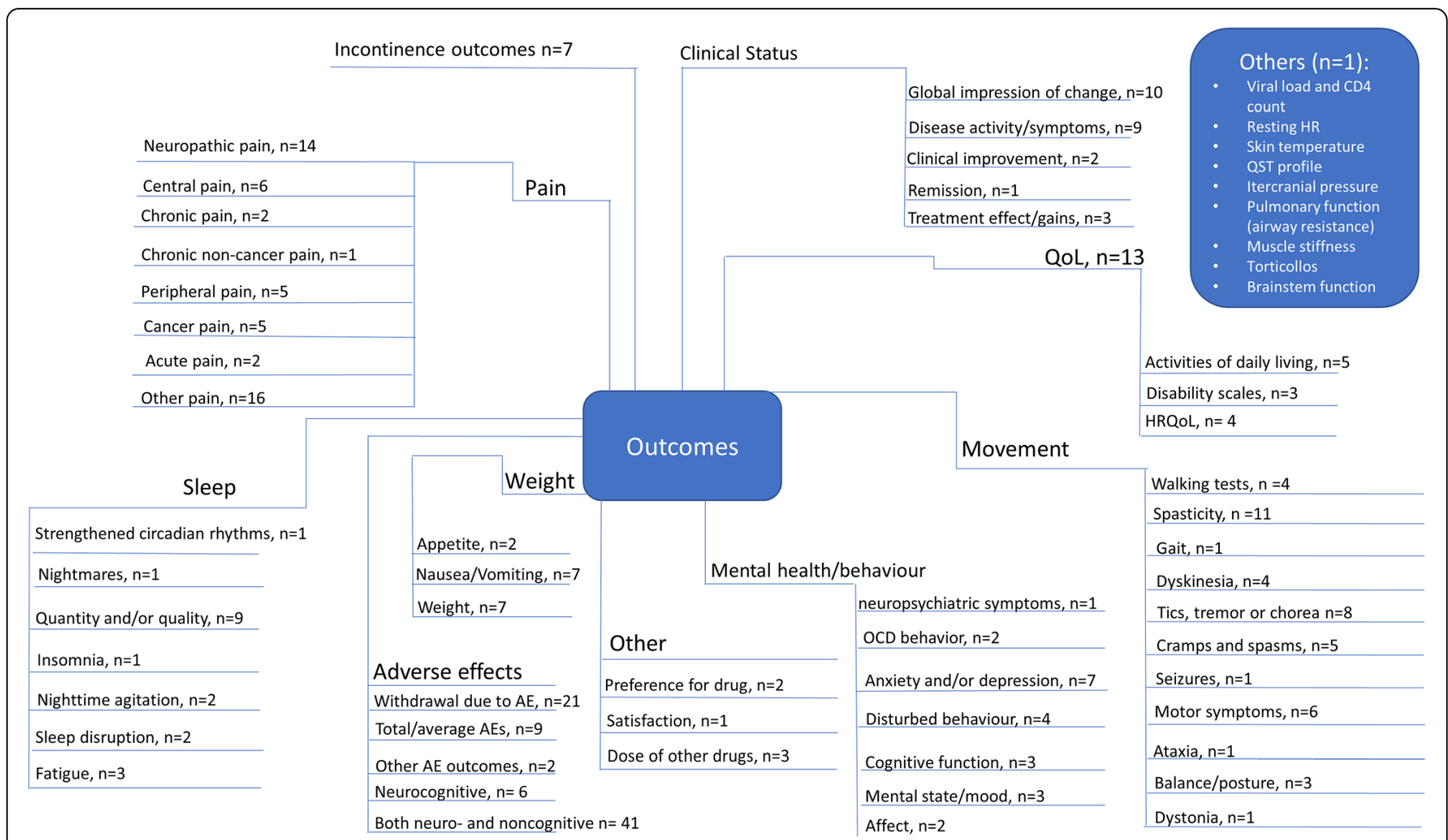

Fig. 5 Outcomes

These reviews covered several different conditions, including injury, chronic pain, rheumatoid arthritis, osteoarthritis, fibromyalgia, HIV/AIDS, cancer, and MS or paraplegia.

When restricting to moderate-to-high-quality reviews, only one review each in multiple sclerosis and HIV/ AIDS with a study-by-study analysis on pain remained. One review on cancer favored cannabis for pain reduction. Findings remained the same for MS or paraplegia and rheumatic disease. No review for injury and paint outcomes was of higher quality.

\section{Non-pain outcomes}

The types of non-pain outcomes included in the reviews varied by condition/illness. The most commonly reported outcomes (see Fig. 5 for overall outcomes) when comparing cannabis to placebo included muscle- or movement-related outcomes ( $n$ $=20)$, quality of life $(n=14)$, and sleep outcomes $(n=10)$.

There was no consistent pattern for non-pain outcomes either within or across medical conditions. Many $(n=24,33 \%)$ reviews assessing non-pain outcomes reported the results of those analyses study-by-study. Conflicting results are observed in some cases due to the use of different measures, such as different ways of quantifying spasticity in patients with multiple sclerosis [56, 91].
One review each addressing neurological conditions [50] (outcome: muscle cramps) and MS/paraplegia [27] (outcomes: spasticity, spasm, cognitive function, daily activities, motricity, and bladder function) showed no difference between groups.

\section{Adverse effects}

Adverse effects were reported in most reviews comparing cannabis with placebo $(49 / 59,83 \%)$. Most adverse events were reported study-by-study, with few reviews $(n=16 / 59,27 \%)$ conducting a narrative or quantitative synthesis. Serious adverse effects were reported in 21/59 (36\%) reviews, and minor adverse effects were reported in 30/59 (51\%) reviews. The remaining reviews did not define the difference between serious and minor adverse events. The most commonly reported serious adverse events included psychotic symptoms $(n=6)$, severe dysphoric reactions $(n=3)$, seizure $(n=3)$, and urinary tract infection $(n=2)$. The most commonly reported minor adverse events included somnolence/drowsiness $(n=$ $28)$, dizziness $(n=27)$, dry mouth $(n=20)$, and nausea $(n=18)$. Many reviews $(n=37 / 59,63 \%)$ comparing cannabis to placebo reported both neurocognitive and non-cognitive adverse effects. Withdrawals due to adverse events were reported in 22 (37\%) reviews. 


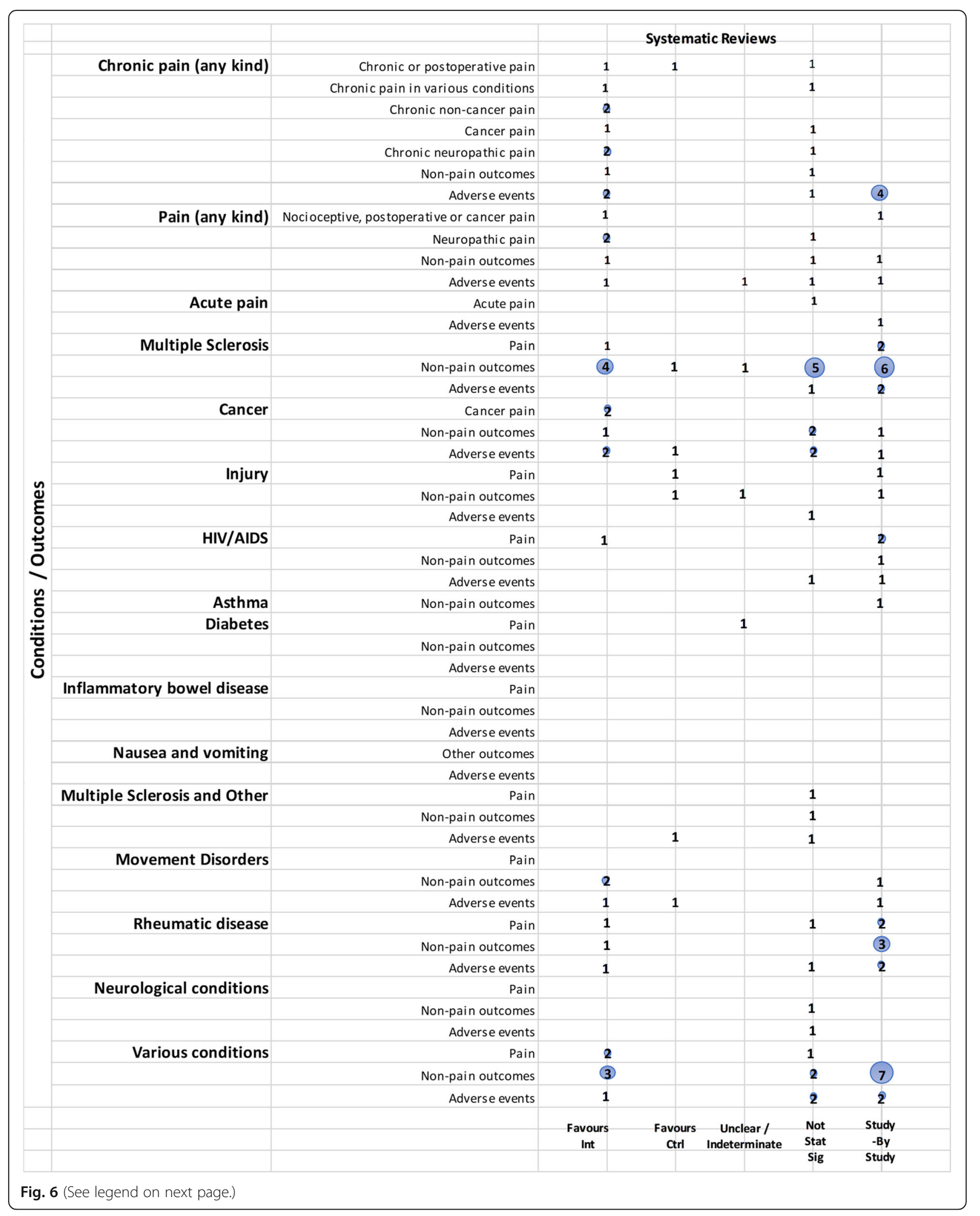


(See figure on previous page.)

Fig. 6 Cannabis vs. placebo. Authors' presentations of the findings were mapped using the categorization shown in Table 2. According to the reviews' intended scope for the condition being treated, outcomes were mapped into "pain," "non-pain outcomes," and "adverse events." For each condition and outcome pair (i.e., each row in the grid), the number of reviews reporting findings is shown according to the results categorization. For pain, reviews numbered in different categories signal discordant findings across those reviews. For non-pain outcomes, reviews presenting findings in the different categories would signal different results for different outcomes, as well as discordant findings within and across reviews. Adverse events are grouped as a whole and "favors intervention" would be interpreted as a decrease in events with cannabis when compared with the control group. Favors int = favors intervention; Favors Ctrl = favors control; Not stat sig = not statistically significant

Of the moderate-/high-quality reviews, adverse effect analyses were reported in reviews on pain, multiple sclerosis, cancer, HIV/AIDS, movement disorders, rheumatic disease, and several other conditions. Two reviews on pain showed fewer adverse events with cannabis for euphoria, events linked to alternations in perception, motor function, and cognitive function, withdrawal due to adverse events, sleep, and dizziness or vertigo $[58,90]$. One review on MS showed that there was no statistically significant difference between cannabis and placebo for adverse effects such as nausea, weakness, somnolence, and fatigue [91], while another review on MS/paraplegia reported fewer events in the placebo group for dizziness, somnolence, nausea, and dry mouth [27]. Within cancer reviews, one review found no statistically significant difference between cannabis and placebo for dysphoria or sedation but reported fewer events with placebo for "feeling high," and fewer events with cannabis for withdrawal due to adverse effects [40]. In rheumatic disease, one review reported fewer total adverse events with cannabis and found no statistically significant difference between cannabis and placebo for withdrawal due to adverse events [51].

\section{Cannabis versus other drugs}

Relatively fewer reviews compared cannabis with active drugs ( $n=23 / 72,32 \%$ ) (Fig. 8). Many of the reviews did not synthesize studies quantitatively, and results were reported study-by-study. The most common conditions in reviews comparing cannabis to active drugs were pain, cancer, and rheumatic disease. Comparators included ibuprofen, codeine, diphenhydramine, amitriptyline, secobarbital, prochlorperazine, domperidone, metoclopramide, amisulpride, neuroleptics, isoproterenol, megestrol acetate, pregabalin, gabapentin, and opioids.

1. Pain outcomes

i. Reviews focused on addressing pain across conditions. When comparing across reviews, a mix of results are observed (see Fig. 8), and some were reported study-by-study. One review found no statistically significant difference between cannabinoids and codeine for nociceptive pain, postoperative pain, and cancer pain [65]. Another review favored "other drugs" (amitriptyline and pregabalin) over cannabinoids for neuropathic pain [90]. The distribution of findings was similar when restricting to moderate-to-high-quality reviews.

ii. Reviews focused on treating a condition or family of related conditions. One review on cancer compared cannabinoids and codeine or secobarbital and reported pain results study-bystudy. Another review on fibromyalgia comparing synthetic cannabinoids with amitriptyline also reported pain data study-by-study [39].

\section{Non-pain outcomes}

Two reviews on cancer favored cannabinoids over active drugs (prochlorperazine, domperidone, metoclopramide, and neuroleptics) for patient preference and anti-emetic efficacy [40,60]. Non-pain outcomes were reported study-by-study for the outcome of sleep in neuropathic pain [90] and rheumatic disease [39, 49]. In a review covering various conditions (pain, MS, anorexia, cancer, and immune deficiency), results were unclear or indeterminate for subjective measures of sleep [46].

\section{Adverse effects}

Adverse effects were reported in 20/24 (83\%) of the reviews comparing cannabis to active drugs, and only $6 / 20$ $(30 \%)$ reported a narrative or quantitative synthesis. Many reviews that reported narrative data did not specify whether adverse effects could be attributed to a placebo or active drug comparator.

Of the moderate-to-high-quality reviews, two pain reviews found no statistically significant difference for cannabis compared to codeine or amitriptyline for withdrawals due to adverse events $[65,90]$. Results from one cancer review were mixed, with fewer adverse events for cannabis (compared to prochlorperazine, domperidone, or metoclopramide) or no difference between groups, depending on the type of subgroup analysis that was conducted [40].

\section{Cannabis + active drugs versus placebo + active drugs}

Two reviews compared cannabis with placebo cannabis in combination with an active drug (opioids and 


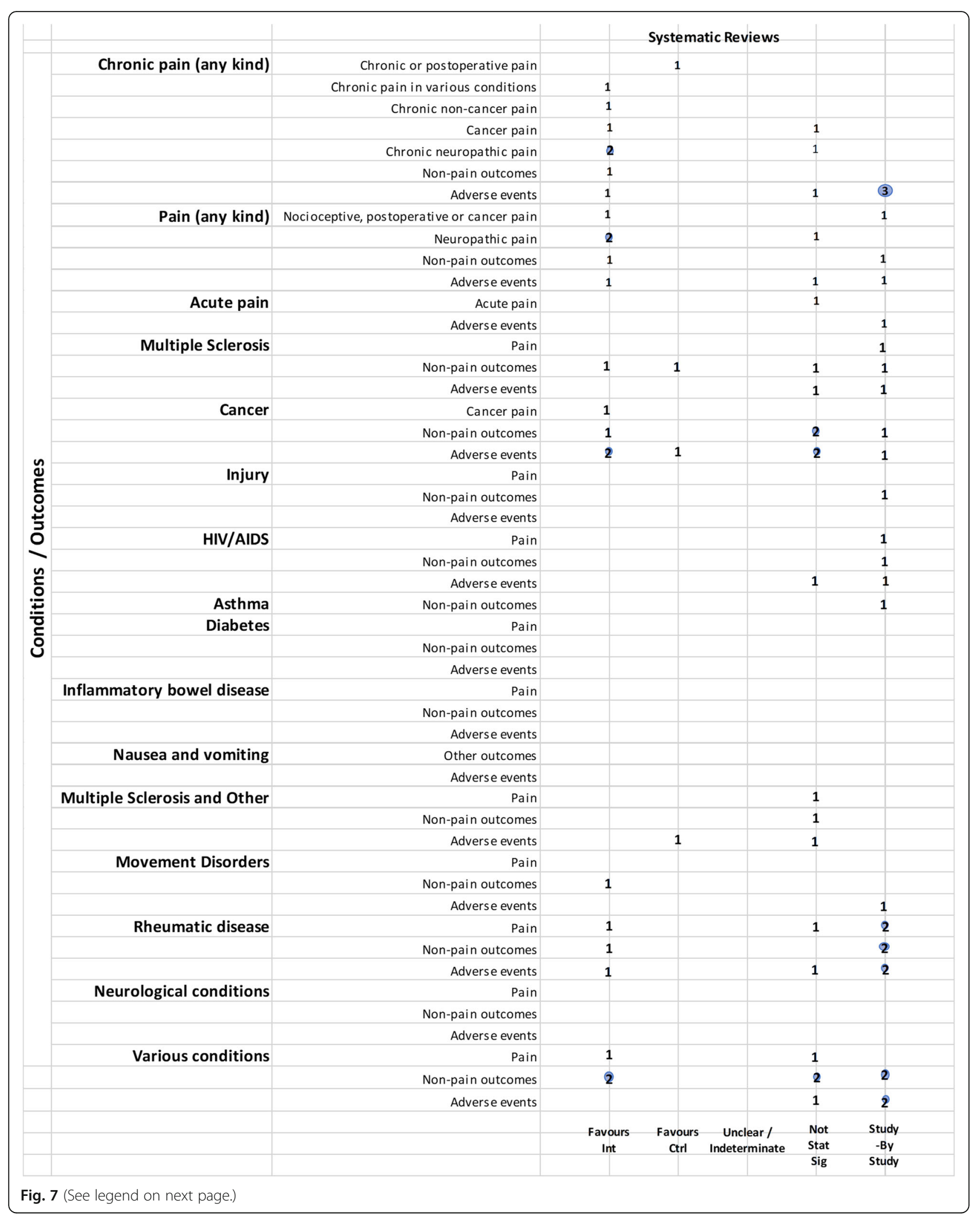


(See figure on previous page.)

Fig. 7 Cannabis vs. placebo, high and moderate quality reviews. Authors' presentations of the findings were mapped using the categorizations shown in Table 2. According to the reviews' intended scope for the condition being treated, outcomes were mapped into "pain," "non-pain outcomes," and "adverse events." For each condition and outcome pair (i.e., each row in the grid), the number of reviews reporting findings is shown according to the results categorization. For pain, reviews numbered in different categories signal discordant findings across those reviews. For non-pain outcomes, reviews presenting findings in the different categories would signal different results for different outcomes, as well as discordant findings within and across reviews. Adverse events are grouped as a whole and "favors intervention" would be interpreted as a decrease in events with cannabis when compared with the control group. Favors int = favors intervention; Favors Ctrl = favors control; Not stat sig = not statistically significant

gabapentin) (Figs. 10 and 11). Both were scored to be of moderate quality. Although one review showed that cannabis plus opioids decreased chronic pain [80], another review on pain in MS included only a single study [81], precluding the ability to determine concordance of results. Cannabis displayed varied effects on non-pain outcomes, including superiority of placebo over cannabis for some outcomes. One review reported withdrawal due to adverse events study-bystudy and also reported that side effects such as nausea, drowsiness, and dizziness were more frequent with higher doses of cannabinoids (data from two included studies) [80].

\section{Cannabis versus other cannabis comparisons}

Six (8\%) reviews compared different cannabis formulations or doses (Figs. 12 and 13). Almost all were reported as study-by-study results, with two reviews including only one RCT. One review for PTSD found only observational data [33] and another review on anxiety and depression combined data from one RCT with cross-sectional study data [19]. A single review on MS reported a narrative synthesis that found a benefit for spasticity. However, it was unclear if the comparator was placebo or THC alone [56]. Four reviews reported adverse effects study-by-study, with a single review comparing side effects from different dosages; in this review, combined extracts of $\mathrm{THC}$ and $\mathrm{CBD}$ were better tolerated than extracts of THC alone [56].

\section{Cannabis versus all comparators}

One review combined all comparators for the evaluation (Fig. 14). The review (combining non-users, placebo and ibuprofen) covered a range of medical conditions and was rated as low quality [30]. No adverse effects were evaluated for this comparison.

\section{Mapping the use of quality assessment and frameworks to interpret the strength of evidence}

Although $83 \%$ of reviews incorporated risk of bias assessments in their interpretation of the evidence, only 11 (15\%) reviews used a framework such as GRADE to evaluate important domains other than risk of bias that would inform the strength of the evidence.

\section{Mapping authors' conclusions or recommendations} Most reviews (43/72 60\%) indicated an inability to draw conclusions, whether due to uncertainty, inconsistent findings, lack of (high quality) evidence, or focusing their conclusion statement on the need for more research. Almost $15 \%$ of reviews (10/72) reported recommendations or conclusions that included some uncertainty. One review (1\%) provided a statement of the extent of the strength of the evidence, which differed according to outcome.

Eleven reviews provided clearer conclusions (14\%). Four indicated that cannabis was not effective or not cost-effective compared to placebo in relation to multiple sclerosis, acute pain, cancer, and injury. Three reviews addressing various conditions provided varying conclusions: one stated cannabis was not effective, one indicated it was modestly safe and effective, and one concluded that cannabis was safe and efficacious as short-term treatment; all reviews were of low quality. The three remaining reviews stated moderate or modest effects for improving chronic pain, compared with placebo or other analgesia; two of those reviews were of medium AMSTAR-2 quality, and one used the GRADE framework for interpreting the strength of the evidence.

The eight remaining included reviews (11\%) did not provide a clear conclusion statement or reported only limitations.

\section{Mapping authors' limitations of the research}

Several of the reviews indicated that few studies, small sample sizes, short duration of treatment, and issues related to outcomes (e.g., definition, timing, and types) were drawbacks to the literature. Some reviews noted methodological issues with and heterogeneity among studies as limitations. A few authors stated that restricting eligibility to randomized trials, English-language studies, or full publications may have affected their review results.

\section{Discussion}

With the increasing use of medical cannabis, an understanding of the landscape of available evidence syntheses is needed to support evidence-informed 


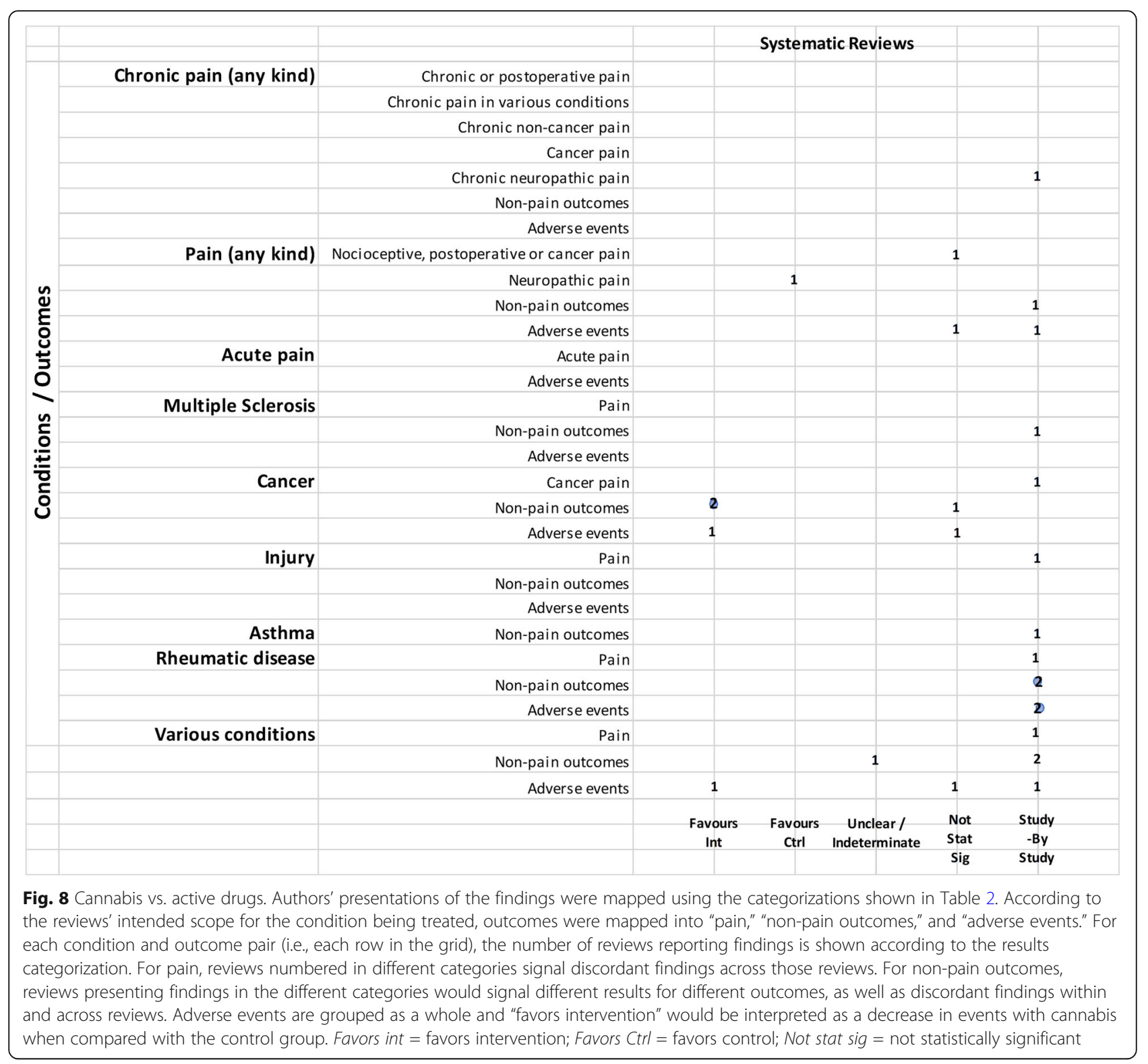

decision-making, policy development, and to inform a research agenda. In this scoping review, we identified 72 systematic reviews evaluating medical cannabis for a range of conditions and illnesses. Half of the reviews were evaluated as being of moderate quality, with only one review scoring high on the AMSTAR-2 assessment tool.

There was disparity in the reported results across reviews, including non-synthesized (study-by-study) data, and many were unable to provide a definitive statement regarding the effectiveness of cannabis (as measured by pain reduction or other relevant outcomes), nor the extent of increased side effects and harms. This is consistent with the limitations declared in general across reviews, such as the small numbers of relevant studies, small sample sizes of individual studies, and methodological weaknesses of available studies. This common theme in review conclusions suggests that while systematic reviews may have been conducted with moderate or high methodological quality, the strength of their conclusions are driven by the availability and quality of the relevant underlying evidence, which was often found to be limited.

Relatively fewer reviews addressed adverse effects associated with cannabis, except to narratively summarize study level data. Although information was provided for placebo-controlled comparisons, none of the comparative effectiveness reviews quantitatively assessed adverse effects data. For the placebocontrolled data, although the majority of adverse effects were mild, the number of reviews reporting 


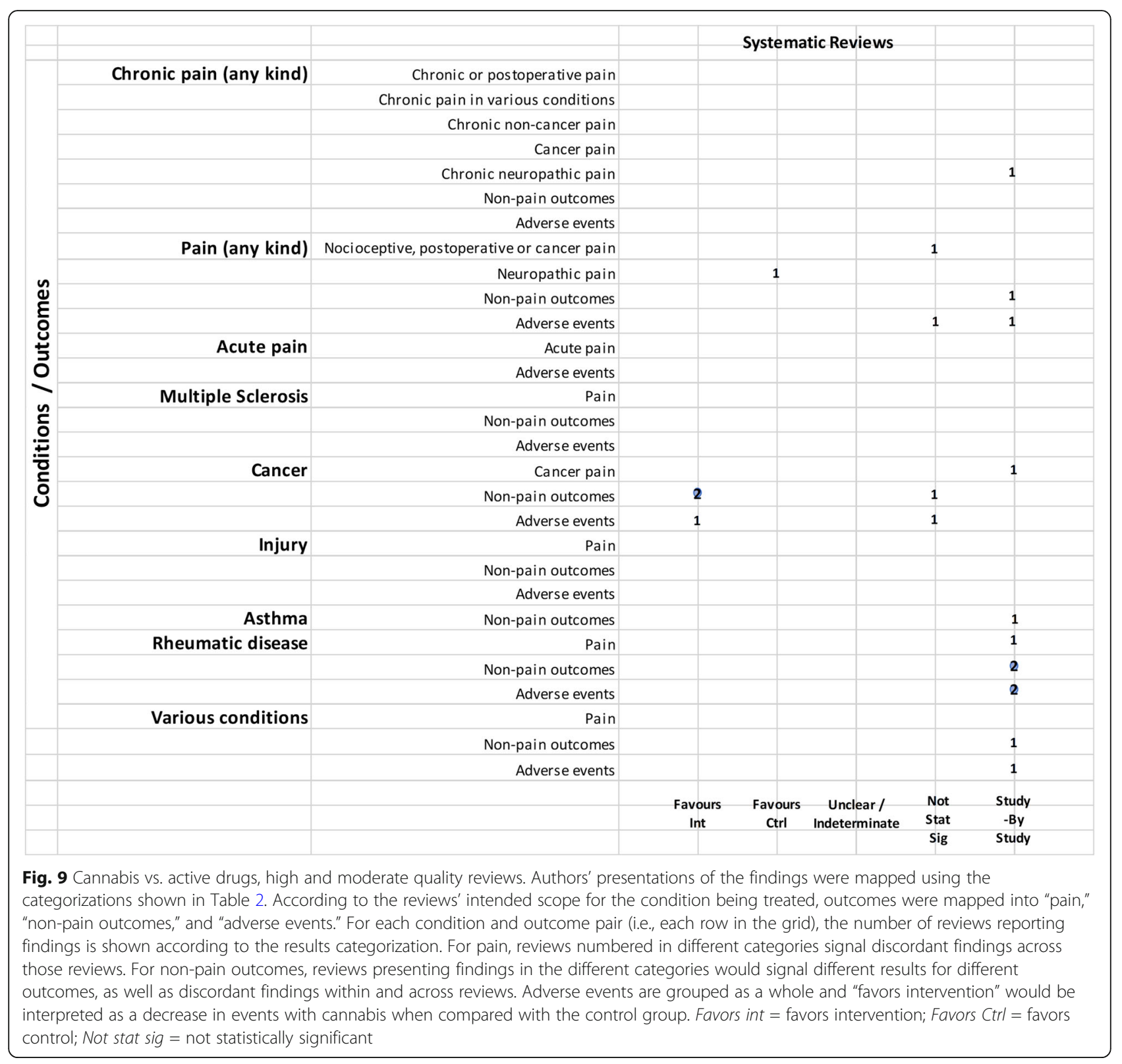

serious adverse effects such as psychotic symptoms $[25,42]$ and suicidal ideation $[68,85]$ warrants caution.

A mix of reviews supporting and not supporting the use of cannabis, according to authors' conclusions, was identified. Readers may wish to consider the quality of the reviews, the use of differing quality assessment tools, additional considerations covered by the GRADE framework, and the potential for spin as possible reasons for these inconsistencies. It is also possible that cannabis has differing effects depending on its type (e.g., synthetic), dose, indication, the type of pain being evaluated (e.g., neuropathic), and the tools used for outcome assessment, which can be dependent on variations in condition. Of potential interest to readers may be a closer examination of the reviews evaluating chronic pain, in order to locate the source(s) of discordance. For example, one review was deemed of moderate quality, used the GRADE framework, and rated the quality of evidence for the effectiveness of cannabis for reducing neuropathic pain as moderate, suggesting that further investigation of cannabis for neuropathic pain may be warranted [80]. The exploration aspects outlined in this paragraph are beyond the purview of scoping review methodology; a detailed assessment of the reviews, including determining the overlap of included studies among similar reviews, potential reasons for the observed discordance of findings, what re-analysis of 


\begin{tabular}{|c|c|c|c|c|c|c|}
\hline \multirow[b]{2}{*}{ Chronic pain (any kind) } & & & \multicolumn{2}{|c|}{ Systematic Reviews } & & \\
\hline & Chronic or postoperative pain & 1 & & & & \\
\hline & Non-pain outcomes & & 1 & 1 & & \\
\hline & Adverse events & & & & \multicolumn{2}{|r|}{1} \\
\hline & & \multirow{2}{*}{$\begin{array}{c}\text { Favours } \\
\text { Int }\end{array}$} & Favours & Unclear / & Not & Study \\
\hline & & & Ctrl & Indeterminate & Stat & -By \\
\hline & & & & & Sig & Study \\
\hline
\end{tabular}

Fig. 10 Cannabis vs. placebo + active drug. Authors' presentations of the findings were mapped using the categorizations shown in Table 2. According to the reviews' intended scope for the condition being treated, outcomes were mapped into "pain," "non-pain outcomes," and "adverse events." For each condition and outcome pair (i.e., each row in the grid), the number of reviews reporting findings is shown according to the results categorization. For pain, reviews numbered in different categories signal discordant findings across those reviews. For non-pain outcomes, reviews presenting findings in the different categories would signal different results for different outcomes, as well as discordant findings within and across reviews. Adverse events are grouped as a whole and "favors intervention" would be interpreted as a decrease in events with cannabis when compared with the control group. Favors int = favors intervention; Favors Ctrl = favors control; Not stat sig = not statistically significant

study-by-study analyses would yield, and an undertaking of missing GRADE assessments would fall outside the bounds of a scoping review and require the use of overview methodology [14].

Our findings are consistent with a recently published summary of cannabis-based medicines for chronic pain management [3]. This report found inconsistent results in systematic reviews of cannabis-based medicines compared to placebo for chronic neuropathic pain, pain management in rheumatic diseases and painful spasms in MS. The authors also concluded that cannabis was not superior to placebo in reducing cancer pain. Four out of eight included reviews scored high on the original AMSTAR tool. The variations between the two tools can be attributed to the differences in our overall assessments. Lastly, the summary report included two reviews that were not located in our original search due to language [93] and the full-text [94] of an abstract [95] that was not located in our search.

This scoping review has identified a plethora of synthesized evidence in relation to medical cannabis. For some conditions, the extent of review replication may be wasteful. Many reviews have stated that additional trials of methodologically robust design and, where possible, of sufficient sample size for precision, are needed to add to the evidence base. This undertaking may require the coordination of multi-center studies to ensure adequate power. Future trials may also help to elucidate the effect of cannabis on different outcomes.

Given authors' reporting of issues in relation to outcomes, future prospective trials should be guided by a standardized, "core" set of outcomes to strive for consistency across studies and ensure relevance to patient-centered care. Development of those core outcomes should be developed using the Core Outcome Measures in Effectiveness Trials (COMET) methodology [96], and further consideration will need to be made in relation to what outcomes may be common across all cannabis research and which outcomes are condition-specific. With maturity of the evidence base, future systematic reviews should seek and include non-journal-published (gray

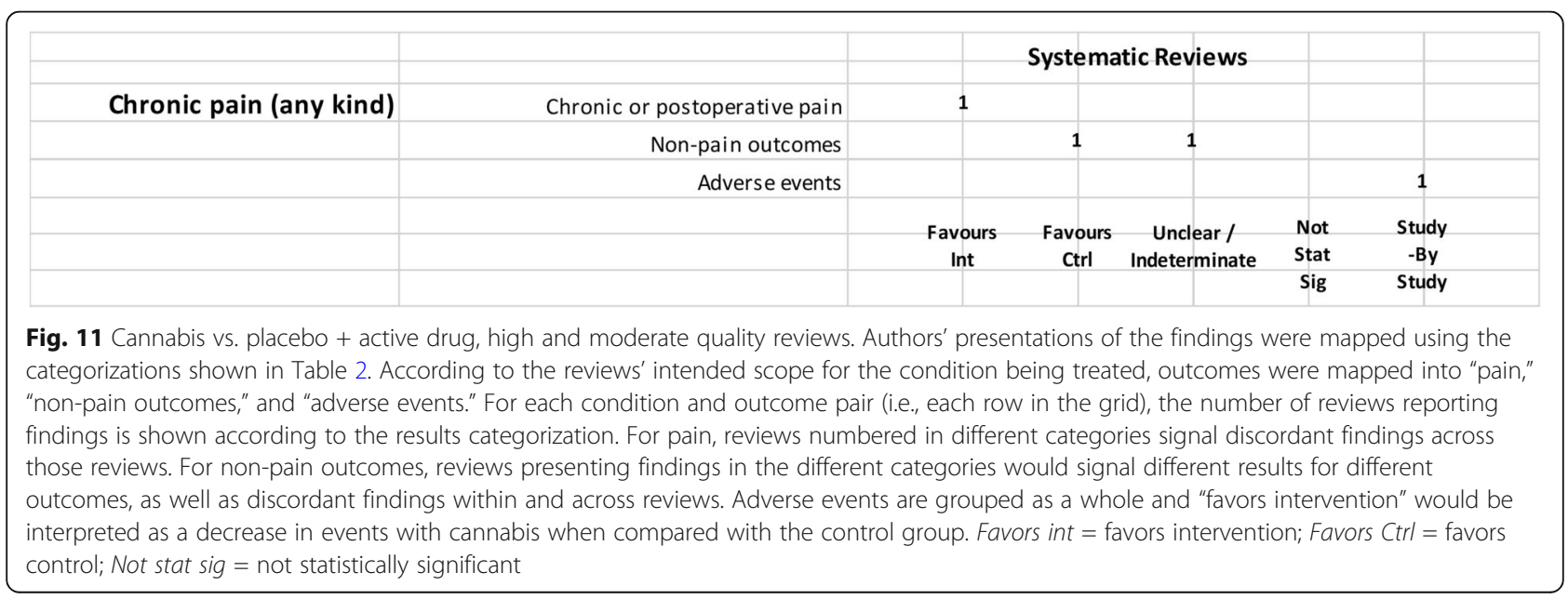




\begin{tabular}{|c|c|c|c|c|c|c|}
\hline \multirow[b]{2}{*}{ Multiple Sclerosis } & \multirow[b]{2}{*}{ Pain } & & \multicolumn{2}{|c|}{ Systematic Reviews } & & \\
\hline & & & & & & \\
\hline & Non-pain outcomes & 1 & & & 1 & 1 \\
\hline & Adverse events & & & & & 1 \\
\hline \multirow[t]{5}{*}{ Asthma } & Non-pain outcomes & & & & & 1 \\
\hline & Adverse events & & & & & 1 \\
\hline & & \multirow{2}{*}{$\begin{array}{c}\text { Favours } \\
\text { Int }\end{array}$} & Favours & & Not & Study \\
\hline & & & Ctrl & Indeterminate & Stat & -By \\
\hline & & & & & Sig & Study \\
\hline
\end{tabular}

Fig. 12 One cannabis formulation vs. other. Authors' presentations of the findings were mapped using the categorizations shown in Table 2. According to the reviews' intended scope for the condition being treated, outcomes were mapped into "pain," "non-pain outcomes," and "adverse events." For each condition and outcome pair (i.e., each row in the grid), the number of reviews reporting findings is shown according to the results categorization. For pain, reviews numbered in different categories signal discordant findings across those reviews. For non-pain outcomes, reviews presenting findings in the different categories would signal different results for different outcomes, as well as discordant findings within and across reviews. Adverse events are grouped as a whole and "favors intervention" would be interpreted as a decrease in events with cannabis when compared with the control group. Favors int = favors intervention; Favors Ctrl = favors control; Not stat sig = not statistically significant

literature) reports and ideally evaluate any nonEnglish-language papers; authors should also adequately assess risk of bias and undertake appropriate syntheses of the literature.

The strengths of this scoping review include the use of an a priori protocol, peer-reviewed search strategies, a comprehensive search for reviews, and consideration of observational designs for adverse effects data. For feasibility, we restricted to English-language reviews, and it is unknown how many of the 39 reviews in other languages that we screened would have met our eligibility criteria. The decision to limit the inclusion of reviews of observational data to adverse effects data was made during the process of full-text screening and for pragmatic reasons. We also did not consider a search of the PROSPERO database for ongoing systematic reviews; however, in preparing this report, we performed a search and found that any completed reviews were already considered for eligibility or were not available at the time of our literature search. When charting results, we took a broad perspective, which may be different than if these reviews were more formally assessed during an overview of systematic reviews.

\section{Conclusions}

Cannabis-based medicine is a rapidly emerging field of study, with implications for both healthcare practitioners and patients. This scoping review is intended to map and collate evidence on the harms and benefits of medical cannabis. Many reviews were unable to provide firm conclusions on the effectiveness of medical cannabis, and results of reviews were mixed. Mild adverse effects

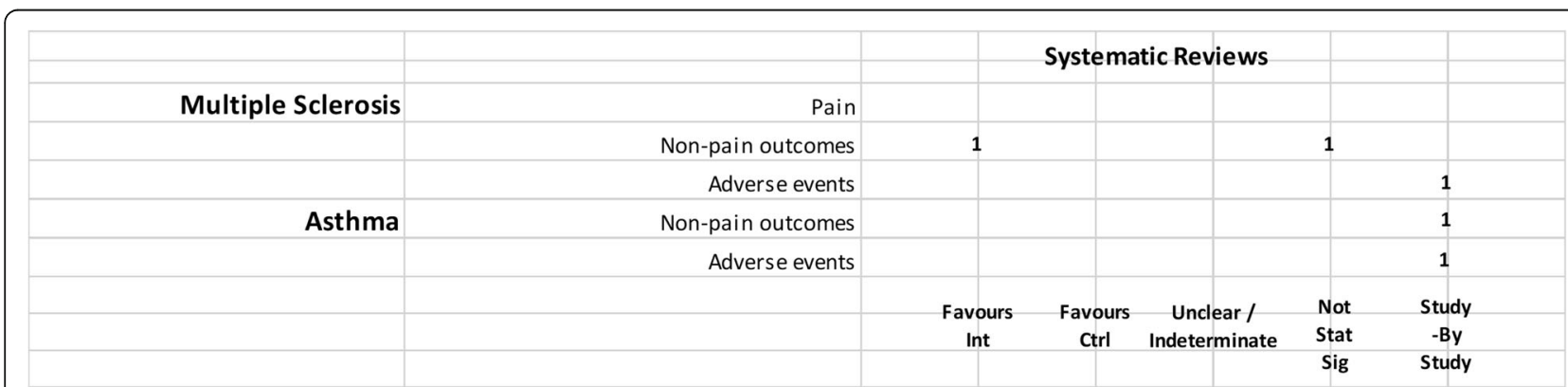

Fig. 13 One cannabis formulation vs. other, high and moderate quality reviews. Authors' presentations of the findings were mapped using the categorizations shown in Table 2. According to the reviews' intended scope for the condition being treated, outcomes were mapped into "pain," "non-pain outcomes," and "adverse events." For each condition and outcome pair (i.e., each row in the grid), the number of reviews reporting findings is shown according to the results categorization. For pain, reviews numbered in different categories signal discordant findings across those reviews. For non-pain outcomes, reviews presenting findings in the different categories would signal different results for different outcomes, as well as discordant findings within and across reviews. Adverse events are grouped as a whole and "favors intervention" would be interpreted as a decrease in events with cannabis when compared with the control group. Favors int = favors intervention; Favors Ctrl = favors control; Not stat sig = not statistically significant 


\begin{tabular}{|c|c|c|c|c|c|c|}
\hline & & & \multicolumn{2}{|c|}{ Systematic Reviews } & & \\
\hline \multirow[t]{6}{*}{ Various conditions } & Pain & & & & & \\
\hline & Non-pain outcomes & & & & \multicolumn{2}{|l|}{1} \\
\hline & Adverse events & & & & \multirow[b]{2}{*}{ Not } & \\
\hline & & \multirow{2}{*}{$\begin{array}{c}\text { Favours } \\
\text { Int }\end{array}$} & Favours & Unclear / & & \\
\hline & & & Ctrl & Indeterminate & \multirow{2}{*}{$\begin{array}{c}\text { Stat } \\
\text { Sig }\end{array}$} & -By \\
\hline & & & & & & Study \\
\hline
\end{tabular}

Fig. 14 Cannabis vs. all comparators combined. Authors' presentations of the findings were mapped using the categorizations shown in Table 2. According to the reviews' intended scope for the condition being treated, outcomes were mapped into "pain," "non-pain outcomes," and "adverse events." For each condition and outcome pair (i.e., each row in the grid), the number of reviews reporting findings is shown according to the results categorization. For pain, reviews numbered in different categories signal discordant findings across those reviews. For non-pain outcomes, reviews presenting findings in the different categories would signal different results for different outcomes, as well as discordant findings within and across reviews. Adverse events are grouped as a whole and "favors intervention" would be interpreted as a decrease in events with cannabis when compared with the control group. Favors int = favors intervention; Favors Ctrl = favors control; Not stat sig = not statistically significant

were frequently but inconsistently reported, and it is possible that harms may outweigh benefits. Evidence from longer-term, adequately powered, and methodologically sound RCTs exploring different types of cannabis-based medicines is required for conclusive recommendations.

\section{Supplementary information}

Supplementary information accompanies this paper at https://doi.org/10. 1186/s13643-019-1243-x

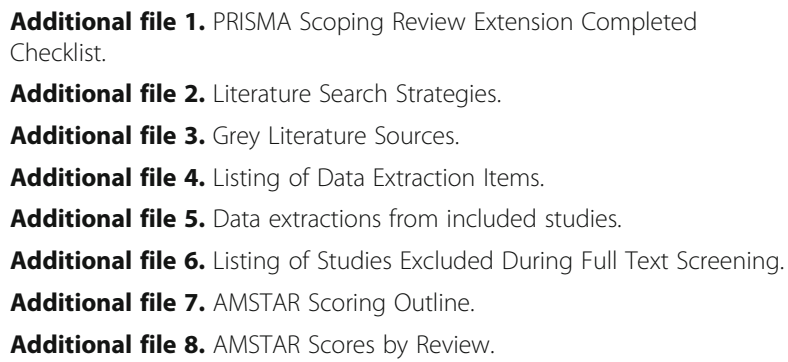

\section{Abbreviations}

CADTH: Canadian Agency for Drugs and Technologies in Health; CAM: Complementary and alternative medicine; CBD: Cannabidiol; GRADE: Grading of Recommendations Assessment, Development and Evaluation; HIV: Human immunodeficiency virus; IBD: Inflammatory bowel disease; MS: Multiple sclerosis; NRS: Numeric rating scale; RCT: Randomized controlled trial; RD: Rheumatic disease; ROB: Risk of bias;

THC: Tetrahydrocannabinol; VAS: Visual analog scale

\section{Acknowledgements}

Not applicable.

\section{Authors' contributions}

$\mathrm{MP}, \mathrm{AS}$, and $\mathrm{BH}$ drafted the initial version of the report. BS designed and implemented the literature search. MP, MT, and CB contributed to review of abstracts and full texts as well as data collection. MP, AS, and BH were responsible for analyses. All authors (MP, AS, MT, CB, BS, SW, MC, SK, BH) contributed to interpretation of findings and revision of drafts and approved the final version of the manuscript.

\section{Funding}

Research reported in this publication was supported by the National Center for Complementary and Integrative Health of the National Institutes of
Health under award number R24AT001293. The content is solely the responsibility of the authors and does not necessarily represent the official views of the National Institutes of Health.

\section{Availability of data and materials}

All data generated or analyzed during this study are included in this published article (and its supplementary information files).

Ethics approval and consent to participate

Not applicable.

\section{Consent for publication}

Not applicable.

\section{Competing interests}

BH has previously received honoraria from Cornerstone Research Group for provision of methodologic advice related to the conduct of systematic reviews and meta-analysis. All other authors declare that they have no conflicts of interest.

\section{Author details}

${ }^{1}$ Knowledge Synthesis Group, Ottawa Methods Centre, Ottawa Hospital Research Institute, The Ottawa Hospital, General Campus, 501 Smyth Road, Ottawa, Ontario K1H 8 L6, Canada. ${ }^{2}$ TRIBE Graduate Program, University of Split School of Medicine, Split, Croatia. ${ }^{3}$ Department of Pharmacology and Therapeutics, McGill University, Montreal, Quebec H3A 2B4, Canada. ${ }^{4}$ Ottawa, Canada. ${ }^{5}$ Center for Integrative Medicine, University of Maryland School of Medicine, Baltimore, MD, USA. ${ }^{6}$ School of Epidemiology and Public Health, University of Ottawa, 451 Smyth Road, Ottawa, Ontario K1H 8 M5, Canada. ${ }^{7}$ Division of Medical Oncology and Department of Medicine, University of Ottawa, Ottawa, Canada. ${ }^{8}$ Department of Pharmacy, The Ottawa Hospital, Ottawa, Canada. ${ }^{9} \mathrm{Clinical}$ Epidemiology Program, The Ottawa Hospital Research Institute, Ottawa, Canada.

Received: 3 June 2019 Accepted: 24 November 2019 Published online: 10 December 2019

\section{References}

1. Watson SJ, Benson JA, Joy JE. Marijuana and medicine: assessing the science base: a summary of the 1999 Institute of Medicine report. Arch Gen Psychiatry. 2000;57:547-52.

2. National Academies of Sciences, Engineering, and Medicine, Health and Medicine Division, Board on Population Health and Public Health Practice \& Committee on the Health Effects of Marijuana: An Evidence Review and Research Agenda. The health effects of cannabis and cannabinoids: the current state of evidence and recommendations for research. US: National Academies Press; 2017.

3. Häuser W, Petzke F, Fitzcharles MA. Efficacy, tolerability and safety of cannabis-based medicines for chronic pain management - an overview of systematic reviews. Eur J Pain Lond Engl. 2018;22:455-70. 
4. Herzog S, et al. Systematic review of the costs and benefits of prescribed cannabis-based medicines for the management of chronic illness: lessons from multiple sclerosis. Pharmacoeconomics. 2017.

5. Aizpurua-Olaizola $\mathrm{O}$, et al. Evolution of the cannabinoid and terpene content during the growth of Cannabis sativa plants from different chemotypes. J. Nat. Prod. 2016;79:324-31.

6. Canadian Agency for Drugs and Technologies in Health. Nabilone for chronic pain management: a review of clinical effectiveness, safety, and guidelines. Rapid Response Rep. 2011.

7. Number of Legal Medical Marijuana Patients - Medical Marijuana - ProCon. org. https://medicalmarijuana.procon.org/view.resource.php?resourcelD=005 889 .

8. Miller, J., December 12, O. C. U. \& 2016. Number of Canadians buying legal medical marijuana triples in past year | Ottawa Citizen. https://ottawacitizen. com/news/local-news/number-of-canadians-buying-legal-medicalmarijuana-triples-in-just-one-year (2016).

9. Colizzi M, Bhattacharyya S. Does cannabis composition matter? Differential effects of delta-9-tetrahydrocannabinol and cannabidiol on human cognition. Curr Addict Rep. 2017:4:62-74.

10. DuPont RL. Examining the debate on the use of medical marijuana. Proc Assoc Am Physicians. 1999;111:166-72.

11. Cohen PJ. Medical marijuana: the conflict between scientific evidence and political ideology. Part one of two. J Pain Palliat Care Pharmacother. 2009; 23:4-25.

12. Cohen PJ. Medical marijuana: the conflict between scientific evidence and political ideology. Part two of two. J Pain Palliat Care Pharmacother. 2009; 23:120-40.

13. Bostwick JM. Blurred boundaries: the therapeutics and politics of medical marijuana. Mayo Clin. Proc. 2012;87:172-86.

14. Arksey H, O'Malley L. Scoping studies: towards a methodological framework. Int J Soc Res Methodol. 2005;8:19-32.

15. Tricco AC, et al. PRISMA Extension for scoping reviews (PRISMA-SCR): Checklist and Explanation. Ann Intern Med. 2018;169:467-73.

16. McGowan J, et al. PRESS peer review of electronic search strategies: 2015 guideline statement. J Clin Epidemiol. 2016;75:40-6.

17. Khangura S, Konnyu K, Cushman R, Grimshaw J, Moher D. Evidence summaries: the evolution of a rapid review approach. Syst Rev. 2012;1:10.

18. Moher D, Liberati A, Tetzlaff J, Altman DG, PRISMA Group. Preferred reporting items for systematic reviews and meta-analyses: the PRISMA statement. Ann Intern Med. 2009:151:264-9, W64.

19. Walsh Z, et al. Medical cannabis and mental health: a guided systematic review. Clin Psychol Rev. 2017:51:15-29.

20. Shea BJ, et al. AMSTAR 2: a critical appraisal tool for systematic reviews that include randomised or non-randomised studies of healthcare interventions, or both. BMJ. 2017;358:j4008.

21. Hsief H, Shannon S. Three approaches to qualitative content analysis. Qual Health Res. 2005;15.

22. Elo S, Kyngas H. The qualitative content analysis process. J Adv Nurs. 2008; 62:107-15.

23. Claflin SB, van der Mei IAF, Taylor BV. Complementary and alternative treatments of multiple sclerosis: a review of the evidence from 2001 to 2016. J Neurol Neurosurg Psychiatr. 2017.

24. Behm K, Morgan P. The effect of symptom-controlling medication on gait outcomes in people with multiple sclerosis: a systematic review. Disabil Rehabil. 2017:1-12.

25. Lim K, See YM, Lee J. A systematic review of the effectiveness of medical cannabis for psychiatric, movement and neurodegenerative disorders. Clin Psychopharmacol Neurosci Off Sci J Korean Coll Neuropsychopharmacol. 2017;15:301-12

26. Aviram J, Samuelly-Leichtag G. Efficacy of cannabis-based medicines for pain management: a systematic review and meta-analysis of randomized controlled trials. Pain Physician. 2017;20:E755-96.

27. da Rovare VP, et al. Cannabinoids for spasticity due to multiple sclerosis or paraplegia: a systematic review and meta-analysis of randomized clinical trials. Complement Ther Med. 2017;34:170-85.

28. Norton C, Czuber-Dochan W, Artom M, Sweeney L, Hart A. Systematic review: interventions for abdominal pain management in inflammatory bowel disease. Aliment Pharmacol Ther. 2017;46:115-25.

29. Snedecor SJ, et al. Systematic review and comparison of pharmacologic therapies for neuropathic pain associated with spinal cord injury. J Pain Res. 2013;6:539-47.
30. Goldenberg M, Reid MW, IsHak WW, Danovitch I. The impact of cannabis and cannabinoids for medical conditions on health-related quality of life: a systematic review and meta-analysis. Drug Alcohol Depend. 2017;174:80-90.

31. Meng H, Johnston B, Englesakis M, Moulin DE, Bhatia A. Selective cannabinoids for chronic neuropathic pain: a systematic review and metaanalysis. Anesth Analg. 2017;125:1638-52.

32. Nugent SM, et al. The effects of cannabis among adults with chronic pain and an overview of general harms: a systematic review. Ann Intern Med. 2017;167:319-31.

33. O'Neil ME, et al. Benefits and harms of plant-based cannabis for posttraumatic stress disorder: a systematic review. Ann Intern Med. 2017; 167:332-40.

34. Abo Youssef $\mathrm{N}$, et al. Cannabinoids for treating neurogenic lower urinary tract dysfunction in patients with multiple sclerosis: a systematic review and meta-analysis. BJU Int. 2017:119:515-21.

35. Mehta S, et al. Systematic review of pharmacologic treatments of pain after spinal cord injury: an update. Arch Phys Med Rehabil. 2016;97:1381-91.

36. Fitzcharles M-A, Baerwald C, Ablin J, Häuser W. Efficacy, tolerability and safety of cannabinoids in chronic pain associated with rheumatic diseases (fibromyalgia syndrome, back pain, osteoarthritis, rheumatoid arthritis): a systematic review of randomized controlled trials. Schmerz Berl Ger. 2016: 30:47-61.

37. Tateo S. State of the evidence: Cannabinoids and cancer pain-a systematic review. J Am Assoc Nurse Pract. 2017;29:94-103.

38. Stevens AJ, Higgins MD. A systematic review of the analgesic efficacy of cannabinoid medications in the management of acute pain. Acta Anaesthesiol Scand. 2017;61:268-80.

39. Walitt B, Klose P, Fitzcharles MA, Phillips T, Hauser W. Cannabinoids for fibromyalgia. Cochrane Database Syst Rev. 2016;7:CD011694.

40. Smith LA, Azariah F, Lavender VTC, Stoner NS, Bettiol S. Cannabinoids for nausea and vomiting in adults with cancer receiving chemotherapy. Cochrane Database Syst Rev. 2015:CD009464.

41. Deshpande A, Mailis-Gagnon A, Zoheiry N, Lakha SF. Efficacy and adverse effects of medical marijuana for chronic noncancer pain: systematic review of randomized controlled trials. Can Fam Physician Med Fam Can. 2015;61: e372-81.

42. Andreae $\mathrm{MH}$, et al. Inhaled cannabis for chronic neuropathic pain: a metaanalysis of individual patient data. J Pain Off J Am Pain Soc. 2015;16:1221-32.

43. Whiting PF, et al. Cannabinoids for medical use: a systematic review and meta-analysis. JAMA. 2015;313:2456-73.

44. Langhorst J, et al. Systematic review of complementary and alternative medicine treatments in inflammatory bowel diseases. J. Crohns Colitis. 2015: 9:86-106.

45. McLoughlin BC, et al. Cannabis and schizophrenia. Cochrane Database Syst Rev. 2014:CD004837.

46. Gates PJ, Albertella L, Copeland J. The effects of cannabinoid administration on sleep: a systematic review of human studies. Sleep Med Rev. 2014;18: 477-87.

47. van den Elsen GAH, et al. Efficacy and safety of medical cannabinoids in older subjects: a systematic review. Ageing Res Rev. 2014;14:56-64.

48. Lutge EE, Gray A, Siegfried N. The medical use of cannabis for reducing morbidity and mortality in patients with HIV/AIDS. Cochrane Database Syst Rev. 2013:CD005175.

49. Fitzcharles M-A, et al. Efficacy, tolerability, and safety of cannabinoid treatments in the rheumatic diseases: a systematic review of randomized controlled trials. Arthritis Care Res. 2016;68:681-8.

50. Baldinger $\mathrm{R}$, Katzberg HD, Weber M. Treatment for cramps in amyotrophic lateral sclerosis/motor neuron disease. Cochrane Database Syst Rev. 2012: CD004157.

51. Richards BL, Whittle SL, Buchbinder R. Neuromodulators for pain management in rheumatoid arthritis. Cochrane Database Syst Rev. 2012;1: CD008921.

52. Lynch ME, Campbell F. Cannabinoids for treatment of chronic non-cancer pain; a systematic review of randomized trials. Br J Clin Pharmacol. 2011;72: 735-44.

53. Phillips TJC, Cherry CL, Cox S, Marshall SJ, Rice ASC. Pharmacological treatment of painful HIV-associated sensory neuropathy: a systematic review and meta-analysis of randomised controlled trials. PloS One. 2010;5:e14433.

54. Phillips RS, et al. Antiemetic medication for prevention and treatment of chemotherapy induced nausea and vomiting in childhood. Cochrane Database Syst Rev. 2010:CD007786 
55. Meyer MJ, et al. Acute management of acquired brain injury part II: an evidence-based review of pharmacological interventions. Brain Inj. 2010;24 706-21.

56. Lakhan SE, Rowland M. Whole plant cannabis extracts in the treatment of spasticity in multiple sclerosis: a systematic review. BMC Neurol. 2009;9:59.

57. Curtis A, Clarke CE, Rickards HE. Cannabinoids for Tourette's syndrome. Cochrane Database Syst Rev. 2009:CD006565.

58. Martin-Sanchez E, Furukawa TA, Taylor J, Martin JL. Systematic review and meta-analysis of cannabis treatment for chronic pain. Pain Med Malden Mass. 2009;10:1353-68.

59. Krishnan S, Cairns R, Howard R. Cannabinoids for the treatment of dementia. Cochrane Database Syst Rev. 2009:CD007204.

60. Machado Rocha FC, Stefano SC, De Cassia Haiek R, Rosa Oliveira LMQ, Da Silveira DX. Therapeutic use of Cannabis sativa on chemotherapy-induced nausea and vomiting among cancer patients: systematic review and metaanalysis. Eur J Cancer Care (Engl). 2008;17:431-43.

61. Wang T, Collet JP, Shapiro S, Ware MA. Adverse effects of medical cannabinoids: a systematic review. Can Med Assoc J. 2008;178:1669-78.

62. Iskedjian M, Bereza B, Gordon A, Piwko C, Einarson TR. Meta-analysis of cannabis based treatments for neuropathic and multiple sclerosis-related pain. Curr Med Res Opin. 2007:23:17-24.

63. Mills RJ, Yap L, Young CA. Treatment for ataxia in multiple sclerosis. Cochrane Database Syst Rev. 2007:CD005029.

64. Shakespeare DT, Boggild M, Young C. Anti-spasticity agents for multiple sclerosis. Cochrane Database Syst Rev. 2003:CD001332

65. Campbell FA, et al. Are cannabinoids an effective and safe treatment option in the management of pain? A qualitative systematic review. BMJ. 2001:323:13-6.

66. Huntley A, Ernst E. Herbal medicines for asthma: a systematic review. Thorax. 2000;55:925-9.

67. Merlin JS, Bulls HW, Vucovich LA, Edelman EJ, Starrels JL. Pharmacologic and nonpharmacologic treatments for chronic pain in individuals with HIV: a systematic review. AIDS Care Psychol Socio Med Asp AIDSHIV. 2016;28:1506-15.

68. Lynch ME, Ware MA. Cannabinoids for the treatment of chronic non-cancer pain: an updated systematic review of randomized controlled trials. J Neuroimmune Pharmacol. 2015;10:293-301.

69. Finnerup NB, et al. Pharmacotherapy for neuropathic pain in adults: a systematic review and meta-analysis. Lancet Neurol. 2015;14:162-73.

70. Snedecor SJ, et al. Systematic review and meta-analysis of pharmacological therapies for painful diabetic peripheral neuropathy. Pain Pract. 2014;14: 167-84.

71. Kuspinar A, Rodriguez AM, Mayo NE. The effects of clinical interventions on health-related quality of life in multiple sclerosis: a meta-analysis. Mult Scler J. 2012;18:1686-704.

72. Gloss D, Vickrey B. Cannabinoids for epilepsy. Cochrane Database Syst Rev Online. 2012:6:CD009270.

73. Macfarlane GJ, et al. Evidence for the efficacy of complementary and alternative medicines in the management of rheumatoid arthritis: a systematic review. Rheumatology. 2011;50:1672-83.

74. Hanson LC, Ersek M, Gilliam R, Carey TS. Oral feeding options for people with dementia: a systematic review. J Am Geriatr Soc. 2011;59:463-72.

75. Mestre T, Ferreira J, Coelho MM, Rosa M, Sampaio C. Therapeutic interventions for symptomatic treatment in Huntington's disease. Cochrane Database Syst Rev. 2009:CD006456.

76. Wheaton P, Mathias JL, Vink R. Impact of early pharmacological treatment on cognitive and behavioral outcome after traumatic brain injury in adults: a meta-analysis. J Clin Psychopharmacol. 2009;29:468-77.

77. Singh $\mathrm{BB}$, et al. Herbal treatments of asthma: a systematic review. J Asthma. 2007:44:685-98

78. Chung $V$, et al. Efficacy and safety of herbal medicines for idiopathic Parkinson's disease: a systematic review. Mov Disord. 2006;21:1709-15.

79. Yavuzsen T, Davis MP, Walsh D, LeGrand S, Lagman R. Systematic review of the treatment of cancer-associated anorexia and weight loss. J Clin Oncol. 2005;23:8500-11.

80. Nielsen $\mathrm{S}$, et al. Opioid-sparing effect of cannabinoids: a systematic review and meta-analysis. Neuropsychopharmacol. 2017;42:1752-65.

81. Canadian Agency for Drugs and Technologies in Health. In: Canadian Agency for Drugs and Technologies in Health, editor. Nabilone for nonchemotherapy associated nausea and weight loss due to medical conditions: a review of the clinical effectiveness and guidelines; 2014.

82. Long-term nabilone use: a review of the clinical effectiveness and safety. Canadian Agency for Drugs and Technologies in Health, 2015.
83. Staples H, Adcock L. Cannabinoids for behavioural symptoms in adults with dementia: a review of clinical effectiveness and guidelines. CADTH. 2018.

84. van den Beuken-van Everdingen $M$, et al. Pharmacological treatment of pain in cancer patients: the role of adjuvant analgesics, a systematic review. Pain Pract Off J World Inst Pain. 2017;17:409-19.

85. Koppel BS, et al. Systematic review: efficacy and safety of medical marijuana in selected neurologic disorders: report of the Guideline Development Subcommittee of the American Academy of Neurology. Neurology. 2014;82: 1556-63.

86. Pringsheim T, et al. Canadian guidelines for the evidence-based treatment of tic disorders: pharmacotherapy. Can J Psychiatry Rev Can Psychiatr. 2012 57:133-43.

87. Scottish Intercollegiate Guidelines Network. Control of pain in adults with cancer: a national clinical guideline. SIGN. 2008.

88. Paice JA, et al. Management of chronic pain in survivors of adult cancers: American Society of Clinical Oncology Clinical Practice Guideline. J Clin Oncol Off J Am Soc Clin Oncol. 2016;34:3325-45.

89. Verleye $L$, et al. Supportive treatment for cancer - part 2: prevention and treatment of adverse events related to chemotherapy and radiotherapy. Good Clin Pract GCP Bruss Belg Health Care Knowl Cent KCE KCE Reports. 2012;191C.

90. Centre for Clinical Practice at NICE (UK). In: National Institute for Health and Care Excellence, editor. Neuropathic pain: the pharmacological management of neuropathic pain in adults in non-specialist settings. UK; 2013.

91. National Clinical Guideline Centre. Multiple sclerosis: management of multiple sclerosis in primary and secondary care. NICE Clinical Guideline. 2014;186.

92. Yadav $\mathrm{V}$, et al. Summary of evidence-based guideline: complementary and alternative medicine in multiple sclerosis: report of the guideline development subcommittee of the American Academy of Neurology. Neurology, 2014;82:1083-92.

93. Mücke $M$, et al. Cannabinoids in palliative care: systematic review and metaanalysis of efficacy, tolerability and safety. Schmerz Berl Ger. 2016;30:25-36.

94. Jawahar R, Oh U, Yang S, Lapane KL. A systematic review of pharmacological pain management in multiple sclerosis. Drugs. 2013:73:1711-22.

95. Jawahar R, Oh U, Yang S, Lapane KL. A systematic review of pharmacological pain management in multiple sclerosis. Database Abstr Rev Eff. 2018;2015

96. Williamson PR, et al. The COMET Handbook: version 1.0. Trials. 2017:18:280.

\section{Publisher's Note}

Springer Nature remains neutral with regard to jurisdictional claims in published maps and institutional affiliations.

Ready to submit your research? Choose BMC and benefit from:

- fast, convenient online submission

- thorough peer review by experienced researchers in your field

- rapid publication on acceptance

- support for research data, including large and complex data types

- gold Open Access which fosters wider collaboration and increased citations

- maximum visibility for your research: over $100 \mathrm{M}$ website views per year

At BMC, research is always in progress.

Learn more biomedcentral.com/submission 